\title{
Nitric Oxide, Oxalic Acid and Hydrogen Peroxide Treatments to Reduce Decay and Maintain Postharvest Quality of 'Valencia' Orange Fruits During Cold Storage
}

\author{
M.A.A. Mohamed ${ }^{*}$, A.F. Abd El-khalek ${ }^{* *}$, H.G. Elmehrat ${ }^{* * *}$ \\ and Gehan. A. Mahmoud* \\ *Fruit Handling Department, Horticulture Research Institute, \\ Agricultural Research Centre, ${ }^{* *}$ Horticulture Department, \\ Faculty of Agricultural, Tanta University and ${ }^{* * *}$ Organic \\ Agricultural, Central Lab., Agricultural Research Centre, \\ Cairo, Egypt
}

\begin{abstract}
7 HE EFFECTIVENESS of postharvest treatments of sodium nitroprusside (SNP), oxalic acid (OA) and hydrogen peroxide $\left(\mathrm{H}_{2} \mathrm{O}_{2}\right)$ treatments and their combinations on postharvest quality and enzyme activity of 'Valencia' orange fruits were examined after harvest in season 2014 and 2015. The experiment were included the following treatments: distilled water (control), $1 \mathrm{mM}$ SNP, $10 \mathrm{mM}$ $\mathrm{OA}, 2 \% \mathrm{H}_{2} \mathrm{O}_{2}, 1 \mathrm{mM} \mathrm{SNP}+10 \mathrm{mM} \mathrm{OA}, 1 \mathrm{mM} \mathrm{SNP}+2 \% \mathrm{H}_{2} \mathrm{O}_{2}$ and $10 \mathrm{mM} \mathrm{OA}+2 \% \mathrm{H}_{2} \mathrm{O}_{2}$ for 5 minutes. All treatments were stored at $8 \pm 1{ }^{\circ} \mathrm{C}$ and $85-90 \%$ relative humidity (RH) followed by one week as a shelf life at ambient temperature $18-23^{\circ} \mathrm{C}$ and $55-65 \% \mathrm{RH}$ for 15 weeks. All postharvst studied maintained postharvest quality of 'Valencia' oranges as compared to untreated fruits (control) during storage. Moreover, combined treatments were more effective than individual treatments. Treated 'Valencia' oranges with $1 \mathrm{mM}$ SNP plus $10 \mathrm{mM} \mathrm{OA}$ or $2 \% \mathrm{H}_{2} \mathrm{O}_{2}$ alleviated decay incidence and reduced weight loss percentage with an increase of marketable fruit percentage. In addition, these applications reduced the activities of polyphenol oxidase (PPO) and pectinase (PE) enzymes, while increased the activity of peroxidase (POX) enzyme. These treatments also decreased loss of firmness, hue angle, lightness values and juice content of fruits. In addition, these applications slowed the increase of soluble solids content (SSC) and SSC/TA ratio as well as decreased loss of titratable acidity (TA) and ascorbic acid contents during cold storage period followed by shelf life. Therefore, the use of postharvest treatments with $1 \mathrm{mM}$ SNP in combination with $10 \mathrm{mM}$ OA or $2 \%$ $\mathrm{H}_{2} \mathrm{O}_{2}$ have good potential strategy to improve the storability and reduce decay incidence as well as maintain postharvest quality of 'Valencia' oranges during cold storage.
\end{abstract}

Keywords: Oxalic acid, Sodium nitroprusside, Hydrogen peroxide, 'Valencia' orange, Fruit quality, Enzyme activity.

Although citrus is considered the greatest planted area among all grown fruits in Egypt, the exported quantity of fresh citrus fruits to foreign markets is restricted. 
Therefore, any effort directed towards keeping fruit quality and decreasing postharvest losses is important to increase the national proceeds. During the last two decades, many investigations were done to try identifying an effective natural disinfecting substance, which may be acceptable to consumers. However, until now there is no alternate applied method.

Postharvest decay is considered the most important factor that limit the shelf life of oranges. Orange fruits are susceptible to a wide variety of fungal diseases. These days, there is a great concern about human health and environmental contamination hazards associated with fungicide residues (Wisniewski \& Wilson, 1992). Thus, there is a stringent need for the development of reliable and environmental friendly methods for protecting perishable crops, particularly fresh fruits, against losses after harvest. Nitric Oxide (NO) is a highly reactive free radical gas engaged in fighting vegetative stress and deterioration of horticultural products. Short term exposure to low doses of NO or its donors can prolong the postharvest life of several fresh fruits and vegetables (Wills et al., 2007 and Zhu \& Zhou, 2007). External postharvest application of NO delayed disease incidence, peel colour changes and reduced activity of softening enzymes (Manjunatha et al., 2010). In addition, application of NO reduced postharvest water loss from horticultural product (Ku et al., 2000). No fumigation reduced the activity of polygalacturonase (PG) enzyme of kiwifruit and peach fruits (Zhu et al., 2010 a, b), banana fruits (Cheng et al., 2009) and Yang et al., 2010). Also, No fumigation reduced the activities of fruits softening enzymes such as exo and endo PG in 'Kensington Pride' mango fruits during cold storage at $13^{\circ} \mathrm{C}$ (Zaharah \& Singh, 2011a, b).

Oxalic acid (OA) is a natural organic acid and playing an important function in systemic resistance and response to environment (Zheng et al., 2012 and Jin et al., 2014). OA application is a secure and hopeful postharvest handling technology for keeping quality and prolonging storage life of fruit (Zheng \& Tian, 2006). OA has shown some antioxidant activities and play a serious function in systemic strength, programmed cell death, redo homeostasis in plants and an anti-senescence effectiveness in harvested fruits (Ding et al., 2007, Zheng et al., 2007a and $\mathrm{Wu}$ et al., 2011). In addition, postharvest treatment of OA reduced the activity of PPO enzyme (Yoruk et al., 2002).

Pre-storage application with $\mathrm{OA}$ enhanced the antioxidant capacities of banana and pomegranate fruits (Sayyari et al., 2010 and Huang et al., 2013a, b). Moreover, OA and oxalate treatments induced systemic resistance against diseases caused by fungi, bacteria and viruses in plants (Mucharromah \& Kuc, 1991 and Toal \& Jones, 1999). Pre-storage application of OA can suppress postharvest disorders and prolong the storage life of mangoes because of delaying the ripening process (Zheng et al., 2007b, c and Zheng et al., 2012). Moreover, postharvest treatment of OA decreased loss of fruit firmness and reduced the activity of exo-PG enzyme beside enhanced the activities of antioxidative enzymes (superoxide dismutase, catalase and peroxidase) (Razzaq 
et al., 2015). Furthermore, who added that, SSC and SSC/TA ratio were lowered, while, TA and ascorbic acid contents were higher in treated fruits as compared to untreated fruits. In addition, postharvest application of bananas with OA maintained peel appearance, prolonged the shelf life of fruits and displayed the potential suitable for commercial application to store the bananas at ambient temperature (Huang et al., $2013 \mathrm{a}, \mathrm{b}$ ).

Postharvest treatment with $\mathrm{H}_{2} \mathrm{O}_{2}$ has been proposed as alternative method to replace currently used chemicals to control postharvest diseases and increasing the storability of fruits and vegetables as well as it pliable as water, surface antiseptic and postharvest aides for organic crops (Suslow, 1997). $\mathrm{H}_{2} \mathrm{O}_{2}$ is an environment friendly compound whose activity is based on oxidation of microbes and it was successfully used to inhibit pathogens of vegetable during storage (Afek et al., 1999). It is now obvious that $\mathrm{H}_{2} \mathrm{O}_{2}$ function as signaling molecules in plants, it is a form of reactive oxygen species (ROS) created because of oxidative stress. Oxidative strain arises from an imbalance in the generation and metabolism of ROS. $\mathrm{H}_{2} \mathrm{O}_{2}$ is one of ROS and used as alternative to chemical materials for disinfecting fruits and vegetables to reduce microbial populations and prolong the shelf life without leaving significant residues or causing loss of postharvest quality (Sapers \& Simmons, 1998 and Sapers et al., 2001). Postharvest treatment with $\mathrm{H}_{2} \mathrm{O}_{2}$ controlled postharvest green mold of lemons (Smilanick et al., 1995). In addition, postharvest applications of $\mathrm{H}_{2} \mathrm{O}_{2}$ of pepper fruits reduced weight loss and rot rate as well as enhanced antioxidants content and improved general appearance during cold storage at $10^{\circ} \mathrm{C}$ for four weeks or during shelf life at $20^{\circ} \mathrm{C}$ for two weeks as compared to untreated fruits (Bayoumi, 2008).

Therefore, the aims of this work were to study the effects of postharvest treatments of 'Valencia' oranges with SNP, OA and $\mathrm{H}_{2} \mathrm{O}_{2}$ alone or in combination on postharvest decay and fruit quality during cold storage.

\section{Materials and Methods}

The present study has been done during two successive seasons 2014 and 2015 on 'Valencia' orange (Citrus sinensis (L.) Osbeck). At maturity stage, 'Valencia' orange fruits were picked randomly from a private orchard at ElBehera Governorate, Egypt. 'Valencia' orange trees were about 23 years old, grafted on 'Volkamariana' rootstock and planted in a sandy soil at 5x5 meters, irrigated by drip system and subjected to all ideal agriculture practices. Fruits were picked directly from similar trees, apparently uniform in size. Fruit were directly transported to postharvest laboratory at Horticulture Research Institute, Agriculture Research Center, Giza governorate. Once arrival to the laboratory, oranges sorted to eliminate defected fruits. Uniform size and appearance orange fruits were washed by tap water then dried by air and held for 24 hours at room temperature. 
Orange fruits were selected and randomly divided into seven groups. Each group included nine boxes consist of 12 individual fruits (108 fruits per treatment, 756 fruits for the whole experiment). Each treatment was immersed for five minutes into one of the following treatments: a- distilled water and used as control, b- $1 \mathrm{mM}$ sodium nitroprusside (SNP), a nitric oxide donor that can release nitric oxide upon in aqueous solvents, c- $10 \mathrm{mM}$ oxalic acid (OX), d- $2 \%$ hydrogen peroxide $\left(\mathrm{H}_{2} \mathrm{O}_{2}\right)$, e - $1 \mathrm{mM} \mathrm{SNP}+10 \mathrm{mM} \mathrm{OX}$, f- $1 \mathrm{mM} \mathrm{SNP}+2 \%$ $\mathrm{H}_{2} \mathrm{O}_{2}$ and g- $10 \mathrm{mM} \mathrm{OX}+2 \% \mathrm{H}_{2} \mathrm{O}_{2}$. Tween-80 at $0.05 \%$ (v/v) was added in each solution to improve wettability and adherence to oranges surface. After immersing treatments, all fruits were dried for one hour at room temperature by electric fan and then packaged in perforated polyethylene bags (five holes of 7 $\mathrm{mm}$ diameter). After that, all treatments were stored at $8 \pm 1^{\circ} \mathrm{C}$ and $85-90 \%$ relative humidity $(\mathrm{RH})$ for 15 weeks. Fruit physical and chemical characteristics were measured at three weeks intervals of cold storage followed by one week at ambient temperature $18-23^{\circ} \mathrm{C}$ and $55-65 \% \mathrm{RH}$ as a shelf life period. The activities of peroxidase (POX), polyphenol oxidase (PPO) and pectinase (PE) enzymes were determined at $0,3,9$ and 15 weeks of storage period followed by one week shelf life at ambient temperature.

\section{Measurements of fruit physical and chemical properties}

Weight loss percentage was calculated by the following equation [(initial fruit weight - fruit weight at examination date) / (initial fruit weight)] $\times 100$.

Decayed fruit percentage was determined by the following equation [(number of decayed fruits at examination date) / (initial number of fruits)] $\times 100$.

Marketable fruit percentage was calculated by the following formula [(sound fruits at examination date) / (initial fruit weight) $] \times 100$.

Fruit colour was measured using a Minolta CR-400 Chroma Meter (Minolta Co. Itd. Osaka, Japan). The measurements of skin colour and gloss were expressed in chromaticity values of hue angle $\left(\mathrm{h}^{\circ}\right)$ and lightness (L), respectively. Three readings were taken at different locations of each orange fruit during each data observation (McGuire, 1992 and Voss, 1992).

Fruit firmness of the peel was assessed by using lfra texture analyzer instrument. The force required to penetrate $1 \mathrm{~cm}$ inside the fruit using a needle probe diameter of $5 \mathrm{~mm}$ was measured. The machine was set with peak mode and speed of $0.3 \mathrm{~mm} / \mathrm{sec}$. Readings were recorded on the two opposite sides of the orange fruit and the results were expressed as the resistance force to the penetrating tester in units of pressure $\mathrm{g} / \mathrm{cm}^{2}$ (Watkins \& Harman, 1981).

Fruit juice content was measured by squeezing six fruits for each treatment represent three replicates and then juice percentage was calculated (w/w). 
Fruit juice content of ascorbic acid (AsA) was determined according to method of adopting the procedure described by AOAC (1990) and was calculated as $\mathrm{mg} / 100 \mathrm{ml}$ juice.

Fruit juice soluble solids content (SSC) was determined by hand refractometer, 0-32 scale (ATAGO N-1 $1_{\mathrm{E}}$, Japan) and expressed in ${ }^{\circ}$ Brix after making the temperature correction at $20^{\circ} \mathrm{C}$ according to AOAC (1990).

Fruit juice content of titratable acidity (TA) was measured by titration as mentioned by AOAC (1990) and was calculated as grams of citric acid/100 ml juice.

Fruit juice SSC/TA ratio was calculated from the values recorded for fruit juice SSC and TA percentages determined.

\section{Enzyme activities}

0.5 gram of fresh orange peel was homogenized by using a mortar and pestle with $0.1 \mathrm{M}$ buffer of phosphate at $4^{\circ} \mathrm{C}(\mathrm{pH}=6.5)$ and stirred for $20 \mathrm{~min}$. The suspension obtained was filtered through one piece of muslin cloth and afterwards centrifuged at $18,000 \times \mathrm{g}$ for 15 minutes, $4^{\circ} \mathrm{C}$. Polyphenol oxidase (PPO) enzyme was measured as mentioned by Fernandez et al. (2011), while peroxidase (POX) and pectinase (PE) enzymes were determined according to Horwitz et al. (1975). The activities of these enzymes were expressed as units per gram fresh weight $\left(\mathrm{U} \mathrm{g}^{-1} \mathrm{fW}\right)$.

\section{Statistical analysis}

This experiment was arranged in a completely randomized design having three replications (Steel et al., 1997) and consisting of two factors (postharvest treatments and storage periods). This experiment was analysis as factorial. Data calculated as percentage were transformed to arcsine of square root before statistical analysis and non-transformed means are shown. The effects of postharvest treatments and cold storage periods on different attributes were analyzed statistically by analysis of variance (ANOVA) using the MSTAT-C statistical package (M-STAT, 1993). Comparisons between means were done by Duncan's multiple range tests (DMRT) at probability $\leq 0.05$.

\section{Results and Discussion}

Influence of postharvest treatments of sodium nitroprusside (SNP), oxalic acid $(\mathrm{OA})$ and hydrogen peroxide $\left(\mathrm{H}_{2} \mathrm{O}_{2}\right)$ solutions on 'Valencia' orange fruit quality during cold storage

Fruit weight loss, decay and marketable percentages

The present study clearly indicated that, weight loss and decay percentages of 'Valencia' orange fruits gradually and significantly increased with prolonging of cold storage period at $8^{\circ} \mathrm{C}$ plus one week as a shelf life at $18-23^{\circ} \mathrm{C}$ in the two 
seasons in this work (Tables 1 and 2). While the percentage of marketable 'Valencia' orange fruits gradually and significantly decreased with prolonging of cold storage period in the two seasons in this study (Table 3).

Data also cleared that, all postharvest treatments significantly decreased the deterioration rate of all these characteristics during cold storage in the two seasons in this experiment as compared with untreated fruits (control). Moreover, it is clear that, postharvest treatment of $1 \mathrm{mM} \mathrm{SNP}$ in combination with $10 \mathrm{mM}$ OA followed by $1 \mathrm{mM}$ SNP in combination with $2 \% \mathrm{H}_{2} \mathrm{O}_{2}$ had the lowest fruit weight loss and decay percentages with the highest marketable fruit percentage as compared to the other postharvest treatments during cold storage in the two seasons in this study.

NO and OA treatments decreased fruit weight loss because of reduced the respiration rate, transpiration through skin, various metabolic activities and inhibited the ethylene production in plums (Singh et al., 2009 and Wu et al., 2011), mangoes (Zheng et al., 2007b, c and Zaharah \& Singh, 2011b) and banana fruits (Huang et al., 2013a).

TABLE 1. Influence of sodium nitroprusside (SNP), oxalic acid (OA) and hydrogen peroxide $\left(\mathrm{H}_{2} \mathrm{O}_{2}\right)$ on weight loss percentage of 'Valencia' oranges during storage at $8^{\circ} \mathrm{C}$ followed by one week shelf life at $18-23^{\circ} \mathrm{C}$

\begin{tabular}{|c|c|c|c|c|c|c|c|c|c|}
\hline \multirow{2}{*}{ Postharvest treatments } & \multicolumn{7}{|c|}{ Storage period (weeks) } & \multirow{2}{*}{\multicolumn{2}{|c|}{ Means }} \\
\hline & & $\mathbf{0}$ & 3 & 6 & 9 & 12 & 15 & & \\
\hline \multicolumn{10}{|c|}{ First Season (2014) } \\
\hline Distilled water (control) & 2.44 & opq & $5.74 \mathrm{i}-1$ & $7.72 \mathrm{fgh}$ & $12.17 \mathrm{bc}$ & $14.72 \mathrm{a}$ & $16.19 \mathrm{a}$ & 9.83 & A \\
\hline $1 \mathrm{mM} \mathrm{SNP}$ & 1.78 & $\mathrm{pq}$ & $4.53 \mathrm{k}-\mathrm{n}$ & $6.96 \mathrm{~g}-\mathrm{j}$ & $7.87 \mathrm{fgh}$ & 9.26 def & $12.94 \mathrm{~b}$ & 7.22 & B \\
\hline $10 \mathrm{mM} \mathrm{OX}$ & 1.63 & $\mathrm{pq}$ & $4.29 \mathrm{lmn}$ & $6.32 \mathrm{~h}-\mathrm{k}$ & 8.23 efg & $10.02 \mathrm{de}$ & $12.35 \mathrm{bc}$ & 7.14 & B \\
\hline $2 \% \mathrm{H}_{2} \mathrm{O}_{2}$ & 1.95 & $\mathrm{pq}$ & $4.65 \mathrm{k}-\mathrm{n}$ & 7.28 ghi & 8.78 efg & 9.40 def & $13.05 \mathrm{~b}$ & 7.52 & B \\
\hline $1 \mathrm{mM} \mathrm{SNP}+10 \mathrm{mM} \mathrm{OX}$ & 1.20 & $\mathrm{q}$ & 3.34 nop & $5.08 \mathrm{k}-\mathrm{n}$ & 7.17 ghi & $8.11 \mathrm{fgh}$ & 9.18 def & 5.68 & $\mathrm{C}$ \\
\hline $1 \mathrm{mM} \mathrm{SNP}+2 \% \mathrm{H}_{2} \mathrm{O}_{2}$ & 1.28 & $\mathrm{q}$ & $3.79 \mathrm{mno}$ & $5.21 \mathrm{j}-\mathrm{m}$ & 7.21 ghi & 8.30 efg & 9.46 def & 5.87 & $\mathrm{C}$ \\
\hline $10 \mathrm{mM} \mathrm{OA}+2 \% \mathrm{H}_{2} \mathrm{O}_{2}$ & 2.00 & $\mathrm{pq}$ & $4.95 \mathrm{k}-\mathrm{n}$ & $6.36 \mathrm{~h}-\mathrm{k}$ & 8.23 efg & 9.47 def & $10.87 \mathrm{~cd}$ & 6.98 & B \\
\hline Means & 1.75 & $\mathrm{~F}$ & $4.47 \mathrm{E}$ & $6.42 \mathrm{D}$ & $8.52 \mathrm{C}$ & $9.90 \mathrm{~B}$ & $12.01 \mathrm{~A}$ & & \\
\hline \multicolumn{10}{|c|}{ Second Season (2015) } \\
\hline Distilled water (control) & 2.27 & $\mathrm{n}$ & $5.22 \mathrm{~lm}$ & $9.05 \mathrm{e}-\mathrm{i}$ & $12.95 \mathrm{~b}$ & $15.59 \mathrm{a}$ & $16.60 \mathrm{a}$ & 10.28 & $\bar{A}$ \\
\hline $1 \mathrm{mM} \mathrm{SNP}$ & 2.13 & $\mathrm{n}$ & $4.85 \mathrm{~lm}$ & $6.62 \mathrm{jkl}$ & $6.26 \mathrm{kl}$ & $10.86 \mathrm{cde}$ & $12.85 \mathrm{~b}$ & 7.26 & $\mathrm{C}$ \\
\hline $10 \mathrm{mM} \mathrm{OX}$ & 2.07 & $\mathrm{n}$ & $5.81 \mathrm{~lm}$ & $6.38 \mathrm{kl}$ & $10.03 \mathrm{ef}$ & $11.88 \mathrm{bcd}$ & $13.29 \mathrm{~b}$ & 8.24 & B \\
\hline $2 \% \mathrm{H}_{2} \mathrm{O}_{2}$ & 2.17 & $\mathrm{n}$ & $5.38 \mathrm{~lm}$ & $7.65 \mathrm{ijk}$ & 10.06 ef & $10.41 \mathrm{de}$ & $12.96 \mathrm{~b}$ & 8.10 & B \\
\hline $1 \mathrm{mM} \mathrm{SNP}+10 \mathrm{mM} \mathrm{OX}$ & 1.52 & $\mathrm{n}$ & $4.29 \mathrm{~m}$ & $5.79 \mathrm{~lm}$ & $7.83 \mathrm{~h}-\mathrm{k}$ & $8.13 \mathrm{~g}-\mathrm{j}$ & 9.83 efg & 6.23 & $\mathrm{D}$ \\
\hline $1 \mathrm{mM} \mathrm{SNP}+2 \% \mathrm{H}_{2} \mathrm{O}_{2}$ & 1.58 & $\mathrm{n}$ & $4.39 \mathrm{~m}$ & $6.10 \mathrm{klm}$ & $8.30 \mathrm{f}-\mathrm{j}$ & $8.30 \mathrm{f}-\mathrm{j}$ & 10.02 ef & 6.45 & $\mathrm{D}$ \\
\hline $10 \mathrm{mM} \mathrm{OA}+2 \% \mathrm{H}_{2} \mathrm{O}_{2}$ & 1.86 & $\mathrm{n}$ & $5.04 \mathrm{~lm}$ & $6.06 \mathrm{klm}$ & 9.57 e-h & $9.22 \mathrm{e}-\mathrm{i}$ & $12.40 \mathrm{bc}$ & 7.36 & $\mathrm{C}$ \\
\hline Means & 1.94 & $\mathrm{~F}$ & $5.00 \mathrm{E}$ & $6.81 \mathrm{D}$ & $9.29 \mathrm{C}$ & $10.63 \mathrm{~B}$ & $12.56 \mathrm{~A}$ & & \\
\hline
\end{tabular}

Means followed by the same letters within postharvest treatments, storage periods and their interactions in each season are not significantly different at level $P \leq 0.05$ according to DMRT.

Egypt. J. Hort. Vol. 43, No. 1 (2016) 
NITRIC OXIDE OXALIC ACID AND HYDROGEN PEROXIDE ...

TABLE 2. Influence of sodium nitroprusside (SNP), oxalic acid (OA) and hydrogen peroxide $\left(\mathrm{H}_{2} \mathrm{O}_{2}\right)$ on decay percentage of 'Valencia' oranges during storage at $8^{\circ} \mathrm{C}$ followed by one week shelf life at $18-23^{\circ} \mathrm{C}$

\begin{tabular}{|c|c|c|c|c|c|c|c|c|c|c|c|c|c|c|}
\hline \multirow{2}{*}{ Postharvest treatments } & \multicolumn{12}{|c|}{ Storage period (weeks) } & \multirow{2}{*}{\multicolumn{2}{|c|}{ Means }} \\
\hline & \multicolumn{2}{|c|}{$\mathbf{0}$} & \multicolumn{2}{|c|}{3} & \multicolumn{2}{|c|}{6} & \multicolumn{2}{|c|}{9} & \multicolumn{2}{|c|}{12} & \multicolumn{2}{|c|}{15} & & \\
\hline \multicolumn{15}{|c|}{ First Season (2014) } \\
\hline Distilled water (control) & 0.00 & $\mathrm{~d}$ & 6.13 & $\mathrm{~cd}$ & 9.88 & bcd & 19.13 & $a b c$ & 26.08 & $\mathrm{ab}$ & 34.08 & a & 15.88 & A \\
\hline $1 \mathrm{mM}$ SNP & 0.00 & d & 0.00 & d & 3.92 & $\mathrm{~cd}$ & 8.88 & bcd & 10.62 & bcd & 12.75 & bcd & 6.03 & $\mathrm{BC}$ \\
\hline $10 \mathrm{mM} \mathrm{OX}$ & 0.00 & d & 0.00 & d & 4.70 & $\mathrm{~cd}$ & 9.68 & bcd & 11.84 & bcd & 14.43 & bcd & 6.78 & $\mathrm{BC}$ \\
\hline $2 \% \mathrm{H}_{2} \mathrm{O}_{2}$ & 0.00 & d & 2.86 & $\mathrm{~cd}$ & 6.76 & $\mathrm{~cd}$ & 11.66 & bcd & 13.22 & bcd & 15.27 & bcd & 8.29 & B \\
\hline $1 \mathrm{mM} \mathrm{SNP}+10 \mathrm{mM} \mathrm{OX}$ & 0.00 & d & 0.00 & $\mathrm{~d}$ & 0.00 & d & 0.00 & d & 2.40 & $\mathrm{~cd}$ & 4.27 & $\mathrm{~cd}$ & 1.11 & $\mathrm{C}$ \\
\hline $1 \mathrm{mM} \mathrm{SNP}+2 \% \mathrm{H}_{2} \mathrm{O}_{2}$ & 0.00 & d & 0.00 & d & 0.00 & $\mathrm{~d}$ & 2.55 & $\mathrm{~cd}$ & 4.08 & $\mathrm{~cd}$ & 9.50 & bcd & 2.69 & $\mathrm{BC}$ \\
\hline $10 \mathrm{mM} \mathrm{OA}+2 \% \mathrm{H}_{2} \mathrm{O}_{2}$ & 0.00 & d & 0.00 & d & 3.14 & $\mathrm{~cd}$ & 5.69 & $\mathrm{~cd}$ & 8.45 & bcd & 11.70 & bcd & 4.83 & $\mathrm{BC}$ \\
\hline Means & 0.00 & $\mathrm{D}$ & 1.28 & $\mathrm{D}$ & 4.06 & $\mathrm{CD}$ & 8.23 & $\mathrm{BC}$ & 10.96 & $\mathrm{AB}$ & 14.57 & A & & \\
\hline \multicolumn{15}{|c|}{ Second Season (2015) } \\
\hline Distilled water (control) & 0.00 & d & 6.66 & $\mathrm{~cd}$ & 11.24 & bcd & 19.61 & abc & 25.36 & $\mathrm{ab}$ & 32.90 & $\mathrm{a}$ & 15.96 & A \\
\hline $1 \mathrm{mM} \mathrm{SNP}$ & 0.00 & $\mathrm{~d}$ & 3.70 & $\mathrm{~cd}$ & 5.10 & $\mathrm{~cd}$ & 7.53 & $\mathrm{~cd}$ & 9.19 & $\mathrm{~cd}$ & 11.40 & bcd & 6.15 & $\mathrm{BC}$ \\
\hline $10 \mathrm{mM} \mathrm{OX}$ & 0.00 & d & 4.15 & $\mathrm{~cd}$ & 6.37 & $\mathrm{~cd}$ & 8.84 & $\mathrm{~cd}$ & 10.94 & bcd & 12.06 & bcd & 7.06 & $\mathrm{BC}$ \\
\hline $2 \% \mathrm{H}_{2} \mathrm{O}_{2}$ & 0.00 & $\mathrm{~d}$ & 4.85 & $\mathrm{~cd}$ & 7.60 & $\mathrm{~cd}$ & 10.59 & bcd & 11.96 & bcd & 13.35 & bcd & 8.06 & B \\
\hline $1 \mathrm{mM} \mathrm{SNP}+10 \mathrm{mM} \mathrm{OX}$ & 0.00 & d & 0.00 & $\mathrm{~d}$ & 0.00 & $\mathrm{~d}$ & 0.00 & d & 3.06 & $\mathrm{~cd}$ & 4.52 & $\mathrm{~cd}$ & 1.26 & $\mathrm{C}$ \\
\hline $1 \mathrm{mM} \mathrm{SNP}+2 \% \mathrm{H}_{2} \mathrm{O}_{2}$ & 0.00 & d & 0.00 & $\mathrm{~d}$ & 0.00 & $\mathrm{~d}$ & 0.00 & d & 4.77 & $\mathrm{~cd}$ & 6.34 & $\mathrm{~cd}$ & 1.85 & $\mathrm{C}$ \\
\hline $10 \mathrm{mM} \mathrm{OA}+2 \% \mathrm{H}_{2} \mathrm{O}_{2}$ & 0.00 & d & 0.00 & $\mathrm{~d}$ & 3.21 & $\mathrm{~cd}$ & 5.17 & $\mathrm{~cd}$ & 7.52 & $\mathrm{~cd}$ & 9.75 & bcd & 4.28 & $\mathrm{BC}$ \\
\hline Means & 0.00 & D & 2.77 & $\mathrm{CD}$ & 4.79 & $\mathrm{CD}$ & 7.39 & $\mathrm{BC}$ & 10.40 & $\mathrm{AB}$ & 12.90 & A & & \\
\hline
\end{tabular}

Means followed by the same letters within postharvest treatments, storage periods and their interactions in each season are not significantly different at level $P \leq 0.05$ according to DMRT.

The safeguard of fruit from infestation of fungal pathogens is largely due to activation of a highly coordinated biochemical and constitutional protection system that helps ward off the dispersal of pathogens (Lawton et al., 1996). Chitinase and $\beta-1,3$-glucanase hydrolyze polymers of fungal dikes walls are thinking to be involved in plant defense mechanisms against fungal infection (Schlumbaum et al., 1986 and Collinge et al., 1993).

NO enhanced fruit resistance to fungal pathogens by inducing the abundance of pathogen related proteins (Kang et al., 2016). OA treatments are contributes to induced systemic resistance in plants by increasing the activity of POX enzyme (Toal and Jones, 1999).

In this study, reduced the decay incidence of 'Valencia' oranges by $1 \mathrm{mM}$ SNP in combination with $10 \mathrm{mM}$ OA treatment may be due to an increase in the activity of POX enzyme and an reduce in the activities of PPO and PE enzymes during storage as mentioned by Tian et al. (2006) and Zheng et al. (2012). 
The reduction of rot rate by using $\mathrm{H}_{2} \mathrm{O}_{2}$ attributed to $\mathrm{H}_{2} \mathrm{O}_{2}$ as a reactive oxygen species (ROS), which play an important role in resistance of plant diseases to infection with pathogens (Bayoumi, 2008).

These results are in harmony with those mentioned by Bayoumi (2008) on pepper fruits, Sayyari et al. (2010) on pomegranates and Razzaq et al. (2015) on mangoes. They mentioned that, postharvest treatments with $\mathrm{NO}, \mathrm{OA}$ and $\mathrm{H}_{2} \mathrm{O}_{2}$ treatments reduced the weight loss percentage during storage.

Moreover, These results are in agreement with the findings of Zheng \& Tian (2006) on litchi fruits, Zheng et al. (2007a) on peach fruits and Zheng et al. (2007b, c), Bayoumi (2008) on pepper fruits and Zheng et al. (2012) on mangoes. They mentioned that, the application of postharvest treatments with $\mathrm{NO}, \mathrm{OA}$ and $\mathrm{H}_{2} \mathrm{O}_{2}$ reduced weight loss and decay incidence of stored fruits.

TABLE 3. Influence of sodium nitroprusside (SNP), oxalic acid (OA) and hydrogen peroxide $\left(\mathrm{H}_{2} \mathrm{O}_{2}\right)$ on marketable percentage of 'Valencia' oranges during storage at $8^{\circ} \mathrm{C}$ followed by one week shelf life at $18-23^{\circ} \mathrm{C}$

\begin{tabular}{|c|c|c|c|c|c|c|c|}
\hline \multirow{2}{*}{ Postharvest treatments } & \multicolumn{6}{|c|}{ Storage period (weeks) } & \multirow[b]{2}{*}{ Means } \\
\hline & $\mathbf{0}$ & 3 & 6 & 9 & 12 & 15 & \\
\hline \multicolumn{8}{|c|}{ First Season (2014) } \\
\hline Distilled water (control) & 97.56 & ab $88.13 \mathrm{a}-\mathrm{j}$ & $82.41 \mathrm{c}-1$ & $68.70 \mathrm{i}-\mathrm{m}$ & $59.21 \mathrm{klm}$ & $49.74 \mathrm{~m}$ & $74.29 \mathrm{D}$ \\
\hline $1 \mathrm{mM}$ SNP & 98.22 & a 95.47 a-e & 89.12 a-i & $83.25 \mathrm{~b}-1$ & $80.12 \mathrm{f}-1$ & $74.32 \mathrm{j}-\mathrm{m}$ & 86.75 BC \\
\hline $10 \mathrm{mM} \mathrm{OX}$ & 98.37 & a 95.71 a-d & 88.98 a-i & $82.09 \mathrm{~d}-1$ & $78.14 \mathrm{~g}-1$ & $73.22 \mathrm{klm}$ & $86.09 \mathrm{C}$ \\
\hline $2 \% \mathrm{H}_{2} \mathrm{O}_{2}$ & 98.05 & a $92.50 \mathrm{a}-\mathrm{g}$ & 85.96 a-1 & $79.57 \mathrm{f}-1$ & $77.38 \mathrm{~h}-1$ & $71.68 \mathrm{~lm}$ & $84.19 \mathrm{CD}$ \\
\hline $1 \mathrm{mM} \mathrm{SNP}+10 \mathrm{mM} \mathrm{OX}$ & 98.80 & a $96.66 \mathrm{abc}$ & 94.92 a-e & 92.83 a-f & 89.49 a-i & $86.55 \mathrm{a}-\mathrm{k}$ & $93.21 \mathrm{~A}$ \\
\hline $1 \mathrm{mM} \mathrm{SNP}+2 \% \mathrm{H}_{2} \mathrm{O}_{2}$ & 98.72 & a 96.21 a-d & 94.79 a-e & $90.25 \mathrm{a}-\mathrm{h}$ & $87.62 \mathrm{a}-\mathrm{j}$ & $81.05 \mathrm{e}-1$ & $91.44 \mathrm{AB}$ \\
\hline $10 \mathrm{mM} \mathrm{OA}+2 \% \mathrm{H}_{2} \mathrm{O}_{2}$ & 98.00 & a 95.05 a-e & 90.50 a-h & $86.07 \mathrm{a}-\mathrm{k}$ & $82.07 \mathrm{~d}-1$ & 77.43 h-1 & $88.19 \mathrm{ABC}$ \\
\hline Means & 98.25 & A $94.25 \mathrm{~A}$ & 89.52 B & $83.25 \mathrm{C}$ & $79.15 \mathrm{C}$ & $73.43 \mathrm{D}$ & \\
\hline \multicolumn{8}{|c|}{ Second Season (2015) } \\
\hline Distilled water (control) & 97.73 & ab 88.12 a-g & $79.71 \mathrm{c}-\mathrm{h}$ & $67.44 \mathrm{fgh}$ & $59.05 \mathrm{hi}$ & $50.50 \mathrm{i}$ & $73.76 \mathrm{C}$ \\
\hline $1 \mathrm{mM} \mathrm{SNP}$ & 97.87 & a 91.44 a-e & $88.28 \mathrm{a}-\mathrm{g}$ & $86.21 \mathrm{a}-\mathrm{g}$ & $79.95 \mathrm{c}-\mathrm{h}$ & 75.74 e-h & $86.58 \mathrm{AB}$ \\
\hline $10 \mathrm{mM} \mathrm{OX}$ & 97.93 & a 90.05 a-g & $87.25 \mathrm{a}-\mathrm{g}$ & $81.13 \mathrm{~b}-\mathrm{h}$ & $77.18 \mathrm{e}-\mathrm{h}$ & $74.65 \mathrm{fgh}$ & 84.70 B \\
\hline $2 \% \mathrm{H}_{2} \mathrm{O}_{2}$ & 97.83 & a 89.77 a-g & 84.76 a-g & $79.35 \mathrm{c}-\mathrm{h}$ & $77.63 \mathrm{~d}-\mathrm{h}$ & $73.69 \mathrm{gh}$ & 83.84 B \\
\hline $1 \mathrm{mM} \mathrm{SNP}+10 \mathrm{mM} \mathrm{OX}$ & 98.48 & a $95.71 \mathrm{abc}$ & $94.21 \mathrm{a}-\mathrm{d}$ & 92.18 a-e & $88.82 \mathrm{a}-\mathrm{g}$ & $85.65 \mathrm{a}-\mathrm{g}$ & $92.51 \mathrm{~A}$ \\
\hline $1 \mathrm{mM} \mathrm{SNP}+2 \% \mathrm{H}_{2} \mathrm{O}_{2}$ & 98.42 & a $95.61 \mathrm{abc}$ & 93.90 a-d & 91.70 a-e & $86.93 \mathrm{a}-\mathrm{g}$ & $83.64 \mathrm{a}-\mathrm{g}$ & $91.70 \mathrm{~A}$ \\
\hline $10 \mathrm{mM} \mathrm{OA}+2 \% \mathrm{H}_{2} \mathrm{O}_{2}$ & 98.14 & a 94.96 abc & 90.73 a-f & $85.27 \mathrm{a}-\mathrm{g}$ & $83.25 \mathrm{a}-\mathrm{g}$ & $77.86 \mathrm{~d}-\mathrm{h}$ & $88.37 \mathrm{AB}$ \\
\hline Means & 98.06 & A $92.24 \mathrm{~B}$ & $88.41 \mathrm{BC}$ & $83.32 \mathrm{CD}$ & $78.97 \mathrm{DE}$ & $74.53 \mathrm{E}$ & \\
\hline
\end{tabular}

Means followed by the same letters within postharvest treatments, storage periods and their interactions in each season are not significantly different at level $P \leq 0.05$ according to DMRT. 
Fruit firmness, gloss (lightness), colour (hue angle) and juice content

Skin colour development represented as hue angle value (greenish yellow, around 75 and yellow, around 60) and gloss represented as lightness. Our results in the present study shown that, fruit firmness, lightness and juice percentage gradually and significantly decreased with prolonging of cold storage period during the two seasons in this investigation. Data also cleared that, fruit peel colour changed directly from greenish yellow to yellow with prolonging of cold storage period at $8^{\circ} \mathrm{C}$ followed by one week as a shelf life at ambient temperature during the two seasons in this study (Tables 4, 5, 6 and 7).

Moreover, the results of our study displayed that, postharvest treatments of 1 mM SNP, $10 \mathrm{mM} \mathrm{OA}$ and $2 \% \mathrm{H}_{2} \mathrm{O}_{2}$ individually or in combination significantly decreased these changes rate in comparison to untreated fruits (control) during the two seasons in this work. Moreover, it is obvious that, postharvest treatment of $1 \mathrm{mM}$ SNP in combination with $10 \mathrm{mM}$ OA had the most effective influence in this aspect followed by $1 \mathrm{mM} \mathrm{SNP}$ in combination with $2 \% \mathrm{H}_{2} \mathrm{O}_{2}$.

TABLE 4. Influence of sodium nitroprusside (SNP), oxalic acid (OA) and hydrogen peroxide $\left(\mathrm{H}_{2} \mathrm{O}_{2}\right)$ on fruit firmness $\left(\mathrm{g} / \mathrm{cm}^{2}\right)$ of 'Valencia' oranges during storage at $8^{\circ} \mathrm{C}$ followed by one week shelf life at $18-23^{\circ} \mathrm{C}$

\begin{tabular}{|c|c|c|c|c|c|c|c|}
\hline \multirow{2}{*}{ Postharvest treatments } & \multicolumn{6}{|c|}{ Storage period (weeks) } & \multirow[b]{2}{*}{ Means } \\
\hline & $\mathbf{0}$ & 3 & 6 & 9 & 12 & 15 & \\
\hline \multicolumn{8}{|c|}{ First Season (2014) } \\
\hline Distilled water (control) & $205.00 \mathrm{ab}$ & $187.00 \mathrm{e}$ & $164.33 \mathrm{k}$ & 152.671 & $136.67 \mathrm{~m}$ & $127.33 \mathrm{n}$ & $162.17 \mathrm{E}$ \\
\hline $1 \mathrm{mM} \mathrm{SNP}$ & $206.67 \mathrm{ab}$ & $203.67 \mathrm{ab}$ & $194.00 \mathrm{~d}$ & 184.33 efg & $174.33 \mathrm{i}$ & $166.00 \mathrm{k}$ & $188.17 \mathrm{BCD}$ \\
\hline $10 \mathrm{mM} \mathrm{OX}$ & $206.33 \mathrm{ab}$ & $203.33 \mathrm{ab}$ & $194.00 \mathrm{~d}$ & 181.33 e-h & $173.67 \mathrm{ij}$ & $164.67 \mathrm{k}$ & $187.22 \mathrm{CD}$ \\
\hline $2 \% \mathrm{H}_{2} \mathrm{O}_{2}$ & $205.67 \mathrm{ab}$ & $202.67 \mathrm{bc}$ & $193.67 \mathrm{~d}$ & $180.33 \mathrm{fgh}$ & $173.33 \mathrm{ij}$ & $163.67 \mathrm{k}$ & $186.56 \mathrm{D}$ \\
\hline $1 \mathrm{mM} \mathrm{SNP}+10 \mathrm{mM} \mathrm{OX}$ & $209.00 \mathrm{a}$ & $206.00 \mathrm{ab}$ & $197.67 \mathrm{~cd}$ & $186.00 \mathrm{ef}$ & 178.67 ghi & $168.33 \mathrm{jk}$ & $190.94 \mathrm{~A}$ \\
\hline $1 \mathrm{mM} \mathrm{SNP}+2 \% \mathrm{H}_{2} \mathrm{O}_{2}$ & $208.67 \mathrm{ab}$ & $205.67 \mathrm{ab}$ & $196.67 \mathrm{~d}$ & 185.67 ef & $177.67 \mathrm{hi}$ & $168.00 \mathrm{jk}$ & $190.39 \mathrm{AB}$ \\
\hline $10 \mathrm{mM} \mathrm{OA}+2 \% \mathrm{H}_{2} \mathrm{O}_{2}$ & $207.67 \mathrm{ab}$ & $204.67 \mathrm{ab}$ & $196.00 \mathrm{~d}$ & 184.33 efg & $177.00 \mathrm{hi}$ & $166.67 \mathrm{k}$ & $189.39 \mathrm{ABC}$ \\
\hline Means & $207.00 \mathrm{~A}$ & $201.86 \mathrm{~B}$ & $190.90 \mathrm{C}$ & $179.24 \mathrm{D}$ & $170.19 \mathrm{E}$ & $160.67 \mathrm{~F}$ & \\
\hline \multicolumn{8}{|c|}{ Second Season (2015) } \\
\hline Distilled water (control) & $204.67 \mathrm{ab}$ & $193.00 \mathrm{fgh}$ & $179.67 \mathrm{k}-\mathrm{n}$ & $166.00 \mathrm{pq}$ & $135.00 \mathrm{r}$ & $126.00 \mathrm{~s}$ & $167.39 \mathrm{D}$ \\
\hline $1 \mathrm{mM} \mathrm{SNP}$ & 206.67 a & $203.67 \mathrm{abc}$ & $196.67 \mathrm{c}-\mathrm{f}$ & $184.33 \mathrm{i}-1$ & $179.00 \mathrm{k}-\mathrm{n}$ & $166.67 \mathrm{pq}$ & $189.50 \mathrm{BC}$ \\
\hline $10 \mathrm{mM} \mathrm{OX}$ & $205.33 \mathrm{ab}$ & 202.33 a-e & $195.67 \mathrm{~d}-\mathrm{g}$ & $184.00 \mathrm{i}-1$ & $177.001-0$ & $165.00 \mathrm{q}$ & $188.22 \mathrm{C}$ \\
\hline $2 \% \mathrm{H}_{2} \mathrm{O}_{2}$ & $205.33 \mathrm{ab}$ & 202.33 a-e & 195.33 efg & $182.33 \mathrm{i}-\mathrm{m}$ & $176.33 \mathrm{mno}$ & $164.33 \mathrm{q}$ & $187.67 \mathrm{C}$ \\
\hline $1 \mathrm{mM} \mathrm{SNP}+10 \mathrm{mM} \mathrm{OX}$ & 209.67 a & 206.67 a & $199.00 \mathrm{~b}-\mathrm{f}$ & 189.00 ghi & $182.67 \mathrm{i}-\mathrm{m}$ & 173.00 nop & $193.33 \mathrm{~A}$ \\
\hline $1 \mathrm{mM} \mathrm{SNP}+2 \% \mathrm{H}_{2} \mathrm{O}_{2}$ & 208.67 a & $205.67 \mathrm{ab}$ & $196.67 \mathrm{c}-\mathrm{f}$ & 187.67 hij & $180.33 \mathrm{j}-\mathrm{n}$ & 171.33 opq & $191.72 \mathrm{AB}$ \\
\hline $10 \mathrm{mM} \mathrm{OA}+2 \% \mathrm{H}_{2} \mathrm{O}_{2}$ & $206.00 \mathrm{ab}$ & 203.00 a-d & 195.33 efg & $185.33 \mathrm{ijk}$ & $179.33 \mathrm{k}-\mathrm{n}$ & $167.67 \mathrm{pq}$ & $189.44 \mathrm{BC}$ \\
\hline Means & $206.62 \mathrm{~A}$ & 202.38 B & $194.05 \mathrm{C}$ & $182.67 \mathrm{D}$ & $172.81 \mathrm{E}$ & $162.00 \mathrm{~F}$ & \\
\hline
\end{tabular}

Means followed by the same letters within postharvest treatments, storage periods and their interactions in each season are not significantly different at level $P \leq 0.05$ according to DMRT. 
TABLE 5. Influence of sodium nitroprusside (SNP), oxalic acid (OA) and hydrogen peroxide $\left(\mathrm{H}_{2} \mathrm{O}_{2}\right)$ on fruit lightness of 'Valencia' oranges during storage at $8^{\circ} \mathrm{C}$ followed by one week shelf life at $18-23^{\circ} \mathrm{C}$

\begin{tabular}{|c|c|c|c|c|c|c|c|}
\hline \multirow{2}{*}{ Postharvest treatments } & \multicolumn{6}{|c|}{ Storage period (weeks) } & \multirow[b]{2}{*}{ Means } \\
\hline & $\mathbf{0}$ & 3 & 6 & 9 & 12 & 15 & \\
\hline \multicolumn{8}{|c|}{ First Season (2014) } \\
\hline Distilled water (control) & 69.51 a-e & 57.01 l-o & 53.77 opq & $51.41 \mathrm{pqr}$ & $48.38 \mathrm{r}$ & $46.61 \mathrm{r}$ & $54.45 \mathrm{E}$ \\
\hline $1 \mathrm{mM}$ SNP & $71.55 \mathrm{abc}$ & 68.55 a-f & $66.27 \mathrm{c}-\mathrm{h}$ & $62.91 \mathrm{~g}-\mathrm{k}$ & $59.49 \mathrm{j}-\mathrm{n}$ & $56.39 \mathrm{~m}-\mathrm{p}$ & $64.19 \mathrm{BCD}$ \\
\hline $10 \mathrm{mM} \mathrm{OX}$ & 71.11 a-d & $68.11 \mathrm{a}-\mathrm{g}$ & $65.79 \mathrm{~d}-\mathrm{i}$ & $62.52 \mathrm{~g}-1$ & 57.91 k-o & 54.47 n-q & $63.32 \mathrm{CD}$ \\
\hline $2 \% \mathrm{H}_{2} \mathrm{O}_{2}$ & 70.56 a-d & $67.56 \mathrm{a}-\mathrm{h}$ & $65.51 \mathrm{~d}-\mathrm{i}$ & $62.51 \mathrm{~g}-1$ & $56.61 \mathrm{mno}$ & $51.17 \mathrm{qr}$ & $62.32 \mathrm{D}$ \\
\hline $1 \mathrm{mM} \mathrm{SNP}+10 \mathrm{mM}$ OX & $72.75 \mathrm{a}$ & 69.75 a-e & 67.99 a-g & $64.72 \mathrm{e}-\mathrm{j}$ & $62.32 \mathrm{~h}-1$ & 60.42 i-m & $66.33 \mathrm{~A}$ \\
\hline $1 \mathrm{mM} \mathrm{SNP}+2 \% \mathrm{H}_{2} \mathrm{O}_{2}$ & 72.75 a & 69.75 a-e & 66.65 b-h & $64.10 \mathrm{e}-\mathrm{j}$ & 60.24 i-m & $59.75 \mathrm{j}-\mathrm{n}$ & $65.54 \mathrm{AB}$ \\
\hline $10 \mathrm{mM} \mathrm{OA}+2 \% \mathrm{H}_{2} \mathrm{O}_{2}$ & $72.22 \mathrm{ab}$ & 69.22 a-e & $66.38 \mathrm{c}-\mathrm{h}$ & $63.53 \mathrm{f}-\mathrm{j}$ & $59.75 \mathrm{j}-\mathrm{n}$ & $59.65 \mathrm{j}-\mathrm{n}$ & $65.13 \mathrm{ABC}$ \\
\hline Means & $71.49 \mathrm{~A}$ & $67.14 \mathrm{~B}$ & $64.62 \mathrm{C}$ & $61.67 \mathrm{D}$ & $57.81 \mathrm{E}$ & $55.49 \mathrm{~F}$ & \\
\hline \multicolumn{8}{|c|}{ Second Season (2015) } \\
\hline Distilled water (control) & 68.72 a-e & $60.75 \mathrm{j}-\mathrm{o}$ & $56.32 \mathrm{pq}$ & $54.05 \mathrm{q}$ & $49.99 \mathrm{r}$ & $45.75 \mathrm{~s}$ & $55.93 \mathrm{C}$ \\
\hline $1 \mathrm{mM}$ SNP & $69.75 \mathrm{abc}$ & $66.42 \mathrm{c}-\mathrm{h}$ & $63.99 \mathrm{f}-\mathrm{k}$ & 61.39 i-o & 59.65 k-p & $57.08 \mathrm{opq}$ & $63.05 \mathrm{~B}$ \\
\hline $10 \mathrm{mM} \mathrm{OX}$ & $69.75 \mathrm{abc}$ & $65.42 \mathrm{c}-\mathrm{i}$ & $63.32 \mathrm{~g}-1$ & $61.05 \mathrm{j}-\mathrm{o}$ & 58.99 l-p & $55.75 \mathrm{pq}$ & $62.38 \mathrm{~B}$ \\
\hline $2 \% \mathrm{H}_{2} \mathrm{O}_{2}$ & 69.09 a-d & $65.09 \mathrm{~d}-\mathrm{j}$ & $62.65 \mathrm{~h}-\mathrm{m}$ & 60.72 j-o & $58.65 \mathrm{~m}-\mathrm{p}$ & $55.42 \mathrm{pq}$ & $61.94 \mathrm{~B}$ \\
\hline $1 \mathrm{mM} \mathrm{SNP}+10 \mathrm{mM} \mathrm{OX}$ & $72.09 \mathrm{a}$ & 69.09 a-d & 67.99 a-f & $65.05 \mathrm{~d}-\mathrm{j}$ & 62.32 h-n & 59.08 1-p & $65.94 \mathrm{~A}$ \\
\hline $1 \mathrm{mM} \mathrm{SNP}+2 \% \mathrm{H}_{2} \mathrm{O}_{2}$ & $71.42 \mathrm{a}$ & 68.42 a-e & 67.99 a-f & $64.39 \mathrm{e}-\mathrm{j}$ & $61.32 \mathrm{i}-\mathrm{o}$ & $58.42 \mathrm{~m}-\mathrm{p}$ & $65.33 \mathrm{~A}$ \\
\hline $10 \mathrm{mM} \mathrm{OA}+2 \% \mathrm{H}_{2} \mathrm{O}_{2}$ & $71.09 \mathrm{ab}$ & 68.09 a-f & 66.99 b-g & $64.05 \mathrm{f}-\mathrm{j}$ & 60.99 j-o & $58.08 \mathrm{n}-\mathrm{q}$ & $64.88 \mathrm{~A}$ \\
\hline Means & $70.27 \mathrm{~A}$ & $66.18 \mathrm{~B}$ & $64.18 \mathrm{C}$ & $61.53 \mathrm{D}$ & $58.84 \mathrm{E}$ & $55.65 \mathrm{~F}$ & \\
\hline
\end{tabular}

Means followed by the same letters within postharvest treatments, storage periods and their interactions in each season are not significantly different at level $P \leq 0.05$ according to DMRT.

Fruit softening is associated with changes in cell wall mechanical strength (Valero \& Serrano, 2010). These results are in conformity with those mentioned by Wu et al. (2011) on plums, Zheng et al. (2012) and Razzaq et al. (2015) on mangoes and Li et al. (2014) on papaya fruits. They reported that, NO and OA reduced cell wall hydrolytic enzymes and consequently decreased loss of firmness of fruits during storage.

Our results are supported by the findings of Zhang et al. (2008) and Singh et al. (2009) in plums, Zaharah \& Singh $(2011 \mathrm{a}, \mathrm{b})$ in mangoes, Cheng et al. (2009), Yang et al. (2010) and Huang et al. (2013a) in bananas and Zhu et al. (2010b) and Kang et al. (2016) in peaches. They mentioned that, NO treatment reduced the activities of hydrolytic enzymes and consequently reduced the deterioration rate of fruit firmness. 
TABLE 6. Influence of sodium nitroprusside (SNP), oxalic acid (OA) and hydrogen peroxide $\left(\mathrm{H}_{2} \mathrm{O}_{2}\right)$ on fruit colour represented as hue angle of 'Valencia' oranges during storage at $8^{\circ} \mathrm{C}$ followed by one week shelf life at $18-23^{\circ} \mathrm{C}$

\begin{tabular}{|c|c|c|c|c|c|c|c|c|c|c|c|c|c|c|}
\hline \multirow{2}{*}{ Postharvest treatments } & \multicolumn{12}{|c|}{ Storage period (weeks) } & \multirow{2}{*}{\multicolumn{2}{|c|}{ Means }} \\
\hline & \multicolumn{2}{|c|}{$\mathbf{0}$} & \multicolumn{2}{|c|}{3} & \multicolumn{2}{|c|}{6} & \multicolumn{2}{|c|}{9} & \multicolumn{2}{|c|}{12} & \multicolumn{2}{|c|}{15} & & \\
\hline \multicolumn{15}{|c|}{ First Season (2014) } \\
\hline Distilled water (control) & 69.76 & a-e & 65.33 & $e-i$ & 60.47 & i-1 & 56.07 & $\operatorname{lm}$ & 52.88 & $\mathrm{mn}$ & 50.59 & $\mathrm{n}$ & 59.18 & $\mathrm{C}$ \\
\hline $1 \mathrm{mM}$ SNP & 71.15 & $a b c$ & 70.15 & a-e & 67.95 & $a-f$ & 63.94 & $\mathrm{f}-\mathrm{k}$ & 61.01 & i-1 & 60.38 & i-1 & 65.76 & B \\
\hline $10 \mathrm{mM} \mathrm{OX}$ & 71.33 & $\mathrm{abc}$ & 70.33 & a-e & 67.61 & $\mathrm{a}-\mathrm{g}$ & 63.81 & $\mathrm{f}-\mathrm{k}$ & 60.80 & $i-1$ & 59.84 & $\mathrm{jkl}$ & 65.62 & $\mathrm{~B}$ \\
\hline $2 \% \mathrm{H}_{2} \mathrm{O}_{2}$ & 70.84 & a-d & 69.84 & a-e & 67.25 & b-h & 62.23 & $\mathrm{~g}-\mathrm{k}$ & 60.25 & i-1 & 59.62 & $\mathrm{kl}$ & 65.01 & B \\
\hline $1 \mathrm{mM} \mathrm{SNP}+10 \mathrm{mM} \mathrm{OX}$ & 72.95 & $\mathrm{a}$ & 71.95 & $a b c$ & 69.53 & a-e & 67.66 & $a-f$ & 65.57 & d-i & 63.48 & $\mathrm{f}-\mathrm{k}$ & 68.52 & A \\
\hline $1 \mathrm{mM} \mathrm{SNP}+2 \% \mathrm{H}_{2} \mathrm{O}_{2}$ & 72.29 & $a b c$ & 71.29 & $\mathrm{abc}$ & 68.87 & $a-f$ & 67.00 & b-h & 65.24 & $e-j$ & 62.15 & h-k & 67.81 & A \\
\hline $10 \mathrm{mM} \mathrm{OA}+2 \% \mathrm{H}_{2} \mathrm{O}_{2}$ & 72.49 & $a b$ & 71.49 & $\mathrm{abc}$ & 68.50 & $a-f$ & 66.80 & $\mathrm{c}-\mathrm{h}$ & 65.15 & $e-j$ & 61.86 & $\mathrm{~h}-\mathrm{k}$ & 67.72 & A \\
\hline \multirow[t]{2}{*}{ Means } & 71.54 & $\mathrm{~A}$ & 70.05 & A & 67.17 & $\mathrm{~B}$ & 63.93 & $\mathrm{C}$ & 61.56 & $\mathrm{D}$ & 59.70 & $\mathrm{E}$ & & \\
\hline & & & & & Secon & d Sea & $\sin (2$ & 015) & & & & & & \\
\hline Distilled water (control) & 68.74 & $a b c$ & 64.43 & $\mathrm{~d}-\mathrm{i}$ & 59.34 & $\mathrm{n}-\mathrm{q}$ & 54.13 & $\mathrm{r}$ & 50.04 & $\mathrm{~s}$ & 49.62 & $\mathrm{~s}$ & 57.72 & $\mathrm{E}$ \\
\hline $1 \mathrm{mM}$ SNP & 68.96 & $\mathrm{abc}$ & 67.96 & $a-d$ & 64.20 & $e-k$ & 63.00 & g-n & 60.24 & $1-q$ & 59.15 & opq & 63.92 & $\mathrm{BCD}$ \\
\hline $10 \mathrm{mM} \mathrm{OX}$ & 69.01 & $\mathrm{ab}$ & 67.72 & a-e & 63.96 & $\mathrm{f}-\mathrm{m}$ & 61.43 & h-o & 60.00 & $\mathrm{n}-\mathrm{q}$ & 57.58 & $\mathrm{pq}$ & 63.28 & $\mathrm{CD}$ \\
\hline $2 \% \mathrm{H}_{2} \mathrm{O}_{2}$ & 68.87 & $a b c$ & 67.54 & $\mathrm{a}-\mathrm{f}$ & 63.12 & g-n & 60.58 & $j-p$ & 59.49 & $\mathrm{n}-\mathrm{q}$ & 56.73 & qr & 62.72 & $\mathrm{D}$ \\
\hline $1 \mathrm{mM} \mathrm{SNP}+10 \mathrm{mM} \mathrm{OX}$ & 71.29 & $\mathrm{a}$ & 70.95 & $\mathrm{a}$ & 67.53 & $a-f$ & 64.33 & $d-j$ & 62.24 & g-o & 61.48 & h-o & 66.30 & $\mathrm{~A}$ \\
\hline $1 \mathrm{mM} \mathrm{SNP}+2 \% \mathrm{H}_{2} \mathrm{O}_{2}$ & 69.96 & $\mathrm{a}$ & 68.96 & $\mathrm{abc}$ & 65.87 & b-g & 64.00 & $\mathrm{f}-1$ & 61.91 & h-o & 60.48 & $\mathrm{k}-\mathrm{p}$ & 65.20 & $\mathrm{AB}$ \\
\hline $10 \mathrm{mM} \mathrm{OA}+2 \% \mathrm{H}_{2} \mathrm{O}_{2}$ & 69.64 & $\mathrm{ab}$ & 68.64 & $\mathrm{abc}$ & 65.21 & $\mathrm{c}-\mathrm{h}$ & 63.01 & g-n & 60.92 & i-p & 60.16 & $\mathrm{~m}-\mathrm{q}$ & 64.60 & $\mathrm{BC}$ \\
\hline Means & 69.50 & A & 68.03 & $\mathrm{~B}$ & 64.18 & $\mathrm{C}$ & 61.50 & D & 59.26 & $\mathrm{E}$ & 57.89 & $\mathrm{~F}$ & & \\
\hline
\end{tabular}

Means followed by the same letters within postharvest treatments, storage periods and their interactions in each season are not significantly different at level $P \leq 0.05$ according to DMRT.

Moreover, these results are in agreement with the outcome of Cheng et al. (2009) and Huang et al. (2013a) in banana fruits and Zaharah \& Singh (2011a, b) in mangoes. They illustrated that, NO and OA delayed colour development by maintained lightness and higher hue angle of the skin during storage. Moreover, they added that, the influence of NO and OA in delaying colour development of fruits may due to suppression of ethylene production and consequently reduced biosynthesis of carotenoids in the skin.

In addition, OA treatment delayed ripening process, reduced the activities of cell wall hydrolytic enzymes in fruits, thus reduced the decline in fruits firmness during storage as reported by Zheng \& Tian (2006) on litchi fruits and Zheng et al. (2007c) and Zheng et al. (2012) on mangoes. 
TABLE 7. Influence of sodium nitroprusside (SNP), oxalic acid (OA) and hydrogen peroxide $\left(\mathrm{H}_{2} \mathrm{O}_{2}\right)$ on juice percentage of 'Valencia' oranges during storage at $8^{\circ} \mathrm{C}$ followed by one week shelf life at $18-23^{\circ} \mathrm{C}$

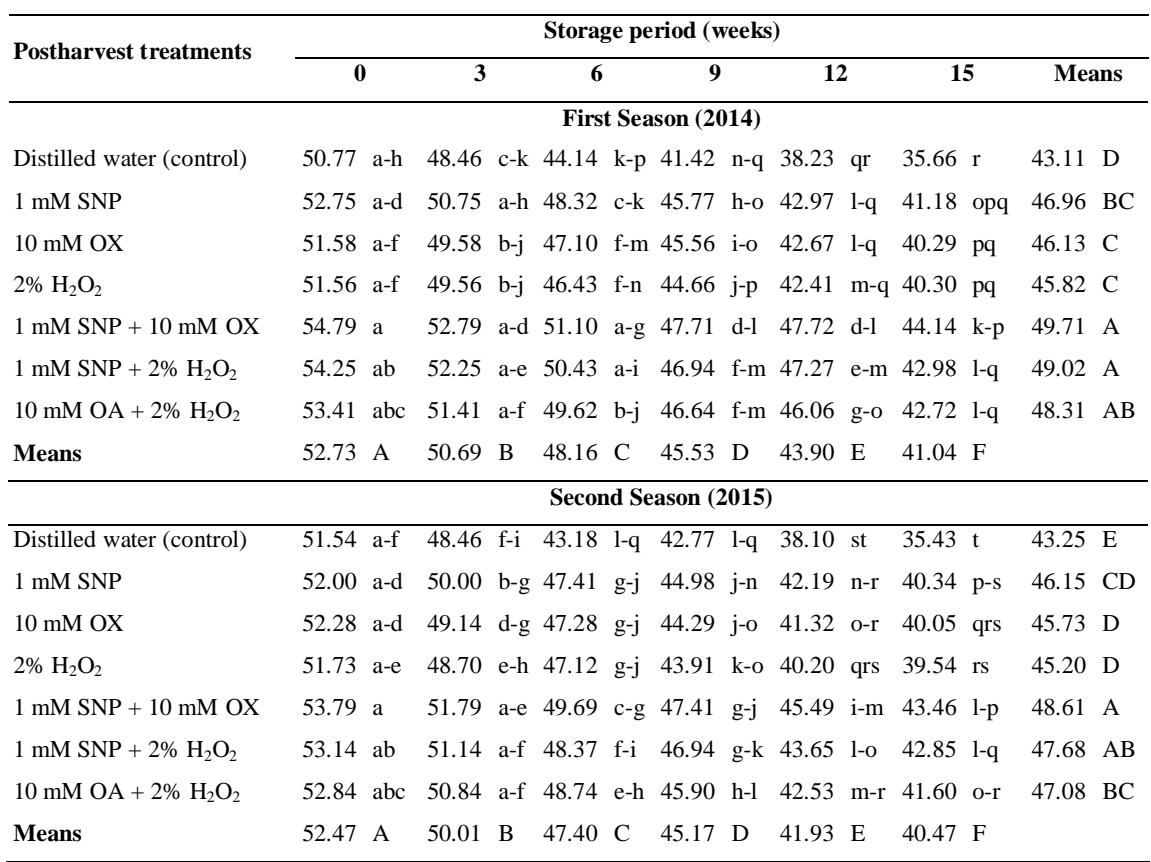

Means followed by the same letters within postharvest treatments, storage periods and their interactions in each season are not significantly different at level $P \leq 0.05$ according to DMRT.

Fruit activities of pectinase (PE), polyphenol oxidase (PPO) and peroxidase (POX) enzymes

The present study cleared that, the activities of POX, PPO and PE enzymes gradually and significantly increased with prolonging of cold storage period in the two seasons in this investigation (Tables 8, 9 and 10).

Moreover, it is obvious that, all postharvest treatments significantly reduced the activities rate of PPO and PE enzymes of 'Valencia' oranges as compared to untreated fruits (control) during cold storage at $8^{\circ} \mathrm{C}$ followed by one week as a shelf life at ambient temperature. On contrary, all postharvest applications significantly increased the activity rate of POX enzyme. In addition, postharvest treatment of $1 \mathrm{mM}$ SNP in combination with $10 \mathrm{mM}$ OA followed by the other two combinations treatments were more effective in reducing the activities of PPO and PE enzymes as well as increasing the activity POX enzyme than individual treatments during storage in the two seasons in this work. 
NITRIC OXIDE OXALIC ACID AND HYDROGEN PEROXIDE ...

TABLE 8. Influence of sodium nitroprusside (SNP), oxalic acid (OA) and hydrogen peroxide $\left(\mathrm{H}_{2} \mathrm{O}_{2}\right)$ on activity of $\mathrm{POX}$ enzyme $\left(\mathrm{U} \mathrm{g}^{-1} \mathrm{fW}\right)$ of 'Valencia' oranges during storage at $8^{\circ} \mathrm{C}$ followed by one week shelf life at $18-23^{\circ} \mathrm{C}$

\begin{tabular}{|c|c|c|c|c|c|c|c|c|}
\hline \multirow{2}{*}{ Postharvest treatments } & \multicolumn{7}{|c|}{ Storage period (weeks) } & \multirow[b]{2}{*}{ Means } \\
\hline & & $\mathbf{0}$ & & 3 & 9 & & 15 & \\
\hline \multicolumn{9}{|c|}{ First Season (2014) } \\
\hline Distilled water (control) & 0.127 & $\mathrm{jk}$ & 0.225 & h-k & $0.239 \mathrm{~h}-\mathrm{k}$ & 0.246 & hij & $0.209 \mathrm{D}$ \\
\hline $1 \mathrm{mM}$ SNP & 0.178 & $\mathrm{ijk}$ & 0.276 & hi & 0.292 ghi & 0.535 & cde & $0.320 \mathrm{C}$ \\
\hline $10 \mathrm{mM} \mathrm{OX}$ & 0.136 & $\mathrm{jk}$ & 0.234 & h-k & 0.287 ghi & 0.521 & cde & $0.294 \mathrm{C}$ \\
\hline $2 \% \mathrm{H}_{2} \mathrm{O}_{2}$ & 0.107 & $\mathrm{k}$ & 0.205 & h-k & $0.207 \mathrm{~h}-\mathrm{k}$ & 0.412 & efg & $0.233 \mathrm{D}$ \\
\hline $1 \mathrm{mM} \mathrm{SNP}+10 \mathrm{mM} \mathrm{OX}$ & 0.262 & hij & 0.627 & $\mathrm{c}$ & $0.767 \mathrm{~b}$ & 1.084 & a & $0.685 \mathrm{~A}$ \\
\hline $1 \mathrm{mM} \mathrm{SNP}+2 \% \mathrm{H}_{2} \mathrm{O}_{2}$ & 0.178 & $\mathrm{ijk}$ & 0.277 & hi & 0.419 ef & 0.819 & $\mathrm{~b}$ & $0.423 \mathrm{~B}$ \\
\hline $10 \mathrm{mM} \mathrm{OA}+2 \% \mathrm{H}_{2} \mathrm{O}_{2}$ & 0.220 & h-k & 0.318 & fgh & $0.490 \mathrm{de}$ & 0.608 & $\mathrm{~cd}$ & 0.409 B \\
\hline Means & 0.173 & $\mathrm{D}$ & 0.309 & $\mathrm{C}$ & $0.386 \mathrm{~B}$ & 0.603 & A & \\
\hline \multicolumn{9}{|c|}{ Second Season (2015) } \\
\hline Distilled water (control) & 0.134 & $\mathrm{mn}$ & 0.232 & ijk & $0.234 \mathrm{ijk}$ & 0.236 & ijk & $0.209 \mathrm{E}$ \\
\hline $1 \mathrm{mM}$ SNP & 0.161 & $\operatorname{lmn}$ & 0.259 & hij & $0.313 \mathrm{gh}$ & 0.542 & $\mathrm{e}$ & $0.319 \mathrm{C}$ \\
\hline $10 \mathrm{mM} \mathrm{OX}$ & 0.137 & $\mathrm{mn}$ & 0.235 & $\mathrm{ijk}$ & $0.309 \mathrm{gh}$ & 0.544 & e & $0.306 \mathrm{C}$ \\
\hline $2 \% \mathrm{H}_{2} \mathrm{O}_{2}$ & 0.106 & $\mathrm{n}$ & 0.204 & $\mathrm{jkl}$ & $0.250 \mathrm{ij}$ & 0.454 & f & $0.254 \mathrm{D}$ \\
\hline $1 \mathrm{mM} \mathrm{SNP}+10 \mathrm{mM} \mathrm{OX}$ & 0.257 & hij & 0.422 & $\mathrm{f}$ & $0.935 \mathrm{~b}$ & 1.162 & $\mathrm{a}$ & $0.694 \mathrm{~A}$ \\
\hline $1 \mathrm{mM} \mathrm{SNP}+2 \% \mathrm{H}_{2} \mathrm{O}_{2}$ & 0.179 & klm & 0.277 & ghi & $0.449 \mathrm{f}$ & 0.858 & $\mathrm{c}$ & $0.441 \mathrm{~B}$ \\
\hline $10 \mathrm{mM} \mathrm{OA}+2 \% \mathrm{H}_{2} \mathrm{O}_{2}$ & 0.221 & $\mathrm{ijk}$ & 0.319 & $\mathrm{~g}$ & $0.540 \mathrm{e}$ & 0.610 & $\mathrm{~d}$ & $0.423 \mathrm{~B}$ \\
\hline Means & 0.171 & $\mathrm{D}$ & 0.278 & $\mathrm{C}$ & $0.433 \mathrm{~B}$ & 0.629 & A & \\
\hline
\end{tabular}

Means followed by the same letters within postharvest treatments, storage periods and their interactions in each season are not significantly different at level $P \leq 0.05$ according to DMRT.

NO positively maintains the balance between the formation and detoxification of ROS ( $\mathrm{Wu}$ et al., 2012). Low doses of NO encouraged the expression or activation of antioxidant enzymes in plants (Leshem et al., 1998). $\mathrm{NO}$ also could detoxify ROS by reacting with $\mathrm{O}_{2}$ and generating peroxynitrite (Crawford, 2006).

Moreover, OA applications are contributes to induced systemic resistance in plants by increasing the activity of POX enzyme (Toal and Jones, 1999). In this experiment, treated fruits by $1 \mathrm{mM}$ SNP in combination with $10 \mathrm{mM} \mathrm{OA}$ increased the activity of POX enzyme and reduced the activities of PPO and PE enzymes of 'Valencia' oranges during storage, thus reduced the decay incidence of fruits.

POX activity produces the oxidative power for transit connecting of proteins and phenylpropanoid radicals generating an encouragement of cell walls against attempted fungal penetration (Yao \& Tian, 2005)

These results were supported by the findings of Tian et al. (2006) on pears, Bayoumi (2008) on peppers, Zheng et al. (2012) on mangoes, Li et al. (2014) on

Egypt. J. Hort. Vol. 43, No. 1 (2016) 
papaya fruits and Wang et al. (2016) on apricots. They reported that, postharvest applications of $\mathrm{NO}, \mathrm{OA}$ and $\mathrm{H}_{2} \mathrm{O}_{2}$ increased the activities of antioxidant enzymes in fruits as compared to control.

These results are supported by the findings of Ueda et al. (2001) and Zaharah \& Singh (2011a, b) on mangoes, Cheng et al. (2009) and Yang et al. (2010) on banana fruits, Zhu et al. (2010a) on kiwifruit fruits and Zhu et al. (2006), Zhu et al. (2010b) and Kang et al. (2016) on peaches. They postulated that, NO reduced the activities of fruits softening enzymes.

In addition, postharvest applications of $\mathrm{OA}$ and oxalate treatments delaying ripening process of mango fruits (Zheng et al., 2007c and Zheng et al., 2012) and bananas (Huang et al., 2013a) by reducing the activities of softening enzymes during storage.

TABLE 9. Influence of sodium nitroprusside (SNP), oxalic acid (OA) and hydrogen peroxide $\left(\mathrm{H}_{2} \mathrm{O}_{2}\right)$ on activity of PPO enzyme $\left(\mathrm{U} \mathrm{g}^{-1} \mathrm{fW}\right)$ of 'Valencia' oranges during storage at $8^{\circ} \mathrm{C}$ followed by one week shelf life at $18-23^{\circ} \mathrm{C}$

\begin{tabular}{|c|c|c|c|c|c|c|c|}
\hline \multirow{2}{*}{ Postharvest treatments } & \multicolumn{6}{|c|}{ Storage period (weeks) } & \multirow[b]{2}{*}{ Means } \\
\hline & & $\mathbf{0}$ & 3 & & 9 & 15 & \\
\hline \multicolumn{8}{|c|}{ First Season (2014) } \\
\hline Distilled water (control) & 0.238 & e & $0.296 \mathrm{~d}$ & 0.468 & $\mathrm{~b}$ & $0.764 \mathrm{a}$ & $0.441 \mathrm{~A}$ \\
\hline $1 \mathrm{mM}$ SNP & 0.091 & ghi & $0.149 \mathrm{fg}$ & 0.166 & $\mathrm{f}$ & $0.363 \mathrm{c}$ & $0.192 \mathrm{BC}$ \\
\hline $10 \mathrm{mM} \mathrm{OX}$ & 0.091 & ghi & $0.149 \mathrm{fg}$ & 0.169 & $\mathrm{f}$ & $0.367 \mathrm{c}$ & $0.194 \mathrm{BC}$ \\
\hline $2 \% \mathrm{H}_{2} \mathrm{O}_{2}$ & 0.092 & ghi & $0.151 \mathrm{fg}$ & 0.221 & $\mathrm{e}$ & $0.367 \mathrm{c}$ & $0.208 \mathrm{~B}$ \\
\hline $1 \mathrm{mM} \mathrm{SNP}+10 \mathrm{mM} \mathrm{OX}$ & 0.091 & ghi & $0.149 \mathrm{fg}$ & 0.120 & $f-i$ & $0.225 \mathrm{e}$ & $0.146 \mathrm{D}$ \\
\hline $1 \mathrm{mM} \mathrm{SNP}+2 \% \mathrm{H}_{2} \mathrm{O}_{2}$ & 0.088 & hi & $0.146 \mathrm{fgh}$ & 0.150 & fg & $0.323 \mathrm{~cd}$ & $0.177 \mathrm{C}$ \\
\hline $10 \mathrm{mM} \mathrm{OA}+2 \% \mathrm{H}_{2} \mathrm{O}_{2}$ & 0.084 & $\mathrm{i}$ & $0.143 \mathrm{f}-\mathrm{i}$ & 0.162 & $\mathrm{f}$ & $0.369 \mathrm{c}$ & $0.190 \mathrm{BC}$ \\
\hline Means & 0.111 & $\mathrm{D}$ & $0.169 \mathrm{C}$ & 0.208 & $\mathrm{~B}$ & $0.397 \mathrm{~A}$ & \\
\hline \multicolumn{8}{|c|}{ Second Season (2015) } \\
\hline Distilled water (control) & 0.245 & $\mathrm{~h}$ & $0.303 \mathrm{~g}$ & 0.441 & $\mathrm{~b}$ & 0.709 a & $0.424 \mathrm{~A}$ \\
\hline $1 \mathrm{mM}$ SNP & 0.091 & $\mathrm{n}$ & 0.1491 & 0.174 & $\mathrm{k}$ & $0.405 \mathrm{c}$ & $0.205 \mathrm{D}$ \\
\hline $10 \mathrm{mM} \mathrm{OX}$ & 0.092 & $\mathrm{n}$ & 0.1501 & 0.205 & $\mathrm{j}$ & $0.416 \mathrm{c}$ & $0.216 \mathrm{C}$ \\
\hline $2 \% \mathrm{H}_{2} \mathrm{O}_{2}$ & 0.111 & $\mathrm{~m}$ & $0.170 \mathrm{k}$ & 0.237 & hi & $0.385 \mathrm{~d}$ & $0.226 \mathrm{~B}$ \\
\hline $1 \mathrm{mM} \mathrm{SNP}+10 \mathrm{mM} \mathrm{OX}$ & 0.099 & $\mathrm{mn}$ & $0.157 \mathrm{kl}$ & 0.149 & 1 & $0.223 \mathrm{i}$ & $0.157 \mathrm{~F}$ \\
\hline $1 \mathrm{mM} \mathrm{SNP}+2 \% \mathrm{H}_{2} \mathrm{O}_{2}$ & 0.092 & $\mathrm{n}$ & 0.1501 & 0.150 & 1 & $0.366 \mathrm{e}$ & $0.190 \mathrm{E}$ \\
\hline $10 \mathrm{mM} \mathrm{OA}+2 \% \mathrm{H}_{2} \mathrm{O}_{2}$ & 0.088 & $\mathrm{n}$ & 0.1461 & 0.169 & $\mathrm{k}$ & $0.349 \mathrm{f}$ & $0.188 \mathrm{E}$ \\
\hline Means & 0.117 & $\mathrm{D}$ & $0.175 \mathrm{C}$ & 0.218 & B & $0.407 \mathrm{~A}$ & \\
\hline
\end{tabular}

Means followed by the same letters within postharvest treatments, storage periods and their interactions in each season are not significantly different at level $P \leq 0.05$ according to DMRT. 
NITRIC OXIDE OXALIC ACID AND HYDROGEN PEROXIDE ...

TABLE 10. Influence of sodium nitroprusside (SNP), oxalic acid (OA) and hydrogen peroxide $\left(\mathrm{H}_{2} \mathrm{O}_{2}\right)$ on activity of $\mathrm{PE}$ enzyme $\left(\mathrm{U} \mathrm{g}^{-1} \mathrm{fW}\right)$ of 'Valencia' oranges during storage at $8^{\circ} \mathrm{C}$ followed by one week shelf life at $18-23^{\circ} \mathrm{C}$

\begin{tabular}{|c|c|c|c|c|c|c|c|c|c|}
\hline \multirow{2}{*}{ Postharvest treatments } & \multicolumn{8}{|c|}{ Storage period (weeks) } & \multirow[b]{2}{*}{ Means } \\
\hline & & $\mathbf{0}$ & & 3 & & 9 & & 15 & \\
\hline \multicolumn{10}{|c|}{ First Season (2014) } \\
\hline Distilled water (control) & 0.161 & gh & 0.179 & ef & 0.286 & $\mathrm{~b}$ & 0.420 & $\mathrm{a}$ & $0.262 \mathrm{~A}$ \\
\hline 1 mM SNP & 0.106 & $1-0$ & 0.124 & jkl & 0.143 & hi & 0.213 & $\mathrm{~d}$ & $0.147 \mathrm{C}$ \\
\hline $10 \mathrm{mM} \mathrm{OX}$ & 0.105 & $1-0$ & 0.124 & jkl & 0.123 & $\mathrm{jkl}$ & 0.242 & $\mathrm{c}$ & $0.149 \mathrm{C}$ \\
\hline $2 \% \mathrm{H}_{2} \mathrm{O}_{2}$ & 0.130 & $\mathrm{ijk}$ & 0.148 & ghi & 0.187 & $\mathrm{e}$ & 0.277 & $\mathrm{~b}$ & $0.185 \mathrm{~B}$ \\
\hline $1 \mathrm{mM} \mathrm{SNP}+10 \mathrm{mM} \mathrm{OX}$ & 0.096 & no & 0.114 & $k-n$ & 0.137 & $\mathrm{ij}$ & 0.133 & ijk & $0.120 \mathrm{E}$ \\
\hline $1 \mathrm{mM} \mathrm{SNP}+2 \% \mathrm{H}_{2} \mathrm{O}_{2}$ & 0.102 & mno & 0.120 & $j-m$ & 0.119 & $\mathrm{j}-\mathrm{m}$ & 0.191 & $\mathrm{e}$ & $0.133 \mathrm{D}$ \\
\hline $10 \mathrm{mM} \mathrm{OA}+2 \% \mathrm{H}_{2} \mathrm{O}_{2}$ & 0.092 & o & 0.110 & $1-0$ & 0.145 & hi & 0.164 & $\mathrm{fg}$ & $0.128 \mathrm{DE}$ \\
\hline Means & 0.113 & $\mathrm{D}$ & 0.131 & $\mathrm{C}$ & 0.163 & $\mathrm{~B}$ & 0.234 & A & \\
\hline \multicolumn{10}{|c|}{ Second Season (2015) } \\
\hline Distilled water (control) & 0.167 & f & 0.185 & $\mathrm{e}$ & 0.282 & $\mathrm{~b}$ & 0.400 & $\mathrm{a}$ & $0.259 \mathrm{~A}$ \\
\hline $1 \mathrm{mM}$ SNP & 0.104 & k-n & 0.122 & h-k & 0.136 & gh & 0.212 & d & $0.144 \mathrm{CD}$ \\
\hline $10 \mathrm{mM} \mathrm{OX}$ & 0.105 & $j-n$ & 0.123 & hij & 0.126 & hi & 0.252 & $\mathrm{c}$ & $0.152 \mathrm{C}$ \\
\hline $2 \% \mathrm{H}_{2} \mathrm{O}_{2}$ & 0.127 & hi & 0.145 & g & 0.184 & ef & 0.251 & $\mathrm{c}$ & $0.177 \mathrm{~B}$ \\
\hline $1 \mathrm{mM} \mathrm{SNP}+10 \mathrm{mM} \mathrm{OX}$ & 0.094 & $\mathrm{mn}$ & 0.112 & $\mathrm{i}-\mathrm{m}$ & 0.120 & h-l & 0.135 & gh & $0.115 \mathrm{~F}$ \\
\hline $1 \mathrm{mM} \mathrm{SNP}+2 \% \mathrm{H}_{2} \mathrm{O}_{2}$ & 0.092 & $\mathrm{n}$ & 0.110 & i-n & 0.145 & $\mathrm{~g}$ & 0.185 & e & $0.133 \mathrm{E}$ \\
\hline $10 \mathrm{mM} \mathrm{OA}+2 \% \mathrm{H}_{2} \mathrm{O}_{2}$ & 0.103 & $\operatorname{lmn}$ & 0.121 & h-l & 0.148 & g & 0.189 & $\mathrm{e}$ & $0.140 \mathrm{DE}$ \\
\hline Means & 0.113 & $\mathrm{D}$ & 0.131 & $\mathrm{C}$ & 0.163 & $\mathrm{~B}$ & 0.232 & A & \\
\hline
\end{tabular}

Means followed by the same letters within postharvest treatments, storage periods and their interactions in each season are not significantly different at level $P \leq 0.05$ according to DMRT.

Fruit juice contents of soluble solids content (SSC), titratable acidity (TA), SSC/TA ratio and ascorbic acid (AsA)

Juice contents of TA and AsA in 'Valencia' orange fruits declined during cold storage at $8^{\circ} \mathrm{C}$ followed by one week as a shelf life at ambient temperature, while an increasing trend in SSC and SSC/TA ratio were recorded. This trend was cleared during the two seasons in this work (Tables 11, 12, 13 and 14).

Data also cleared that, all postharvest treatments especially in combinations significantly reduced these deterioration in 'Valencia' orange fruits during the two seasons in this work in comparison to untreated fruits, except in the case of SSC, which was insignificant during the first season. 
TABLE 11. Influence of sodium nitroprusside (SNP), oxalic acid (OA) and hydrogen peroxide $\left(\mathrm{H}_{2} \mathrm{O}_{2}\right)$ on SSC ( $\left({ }^{\circ} \mathrm{Brix}\right)$ of 'Valencia' oranges during storage at $8^{\circ} \mathrm{C}$ followed by one week shelf life at $18-23^{\circ} \mathrm{C}$

\begin{tabular}{|c|c|c|c|c|c|c|c|c|c|c|c|c|c|c|}
\hline \multirow{2}{*}{ Postharvest treatments } & \multicolumn{12}{|c|}{ Storage period (weeks) } & \multirow{2}{*}{\multicolumn{2}{|c|}{ Means }} \\
\hline & & $\mathbf{0}$ & & 3 & 6 & & 9 & & 1 & & 15 & & & \\
\hline \multicolumn{15}{|c|}{ First Season (2014) } \\
\hline Distilled water (control) & 9.87 & ef & 9.93 & def & 10.03 & $b-f$ & 10.13 & $a-f$ & 10.47 & $a b$ & 10.50 & $\mathrm{ab}$ & 10.16 & A \\
\hline $1 \mathrm{mM} \mathrm{SNP}$ & 9.70 & $\mathrm{f}$ & 9.90 & ef & 10.03 & b-f & 10.20 & a-e & 10.40 & $a b c$ & 10.43 & $\mathrm{ab}$ & 10.11 & A \\
\hline $10 \mathrm{mM} \mathrm{OX}$ & 9.77 & ef & 9.97 & $c-f$ & 10.13 & $a-f$ & 10.20 & a-e & 10.43 & $a b$ & 10.50 & $\mathrm{ab}$ & 10.17 & A \\
\hline $2 \% \mathrm{H}_{2} \mathrm{O}_{2}$ & 9.77 & ef & 9.97 & $c-f$ & 10.20 & a-e & 10.13 & $a-f$ & 10.47 & $a b$ & 10.50 & $\mathrm{ab}$ & 10.17 & A \\
\hline $1 \mathrm{mM} \mathrm{SNP}+10 \mathrm{mM} \mathrm{OX}$ & 9.70 & $f$ & 9.90 & ef & 10.13 & $a-f$ & 10.20 & a-e & 10.43 & $a b$ & 10.57 & a & 10.16 & A \\
\hline $1 \mathrm{mM} \mathrm{SNP}+2 \% \mathrm{H}_{2} \mathrm{O}_{2}$ & 9.77 & ef & 9.97 & $c-f$ & 10.10 & $a-f$ & 10.20 & a-e & 10.37 & a-d & 10.50 & $\mathrm{ab}$ & 10.15 & A \\
\hline $10 \mathrm{mM} \mathrm{OA}+2 \% \mathrm{H}_{2} \mathrm{O}_{2}$ & 9.77 & ef & 9.97 & $c-f$ & 10.17 & $a-f$ & 10.17 & $a-f$ & 10.47 & $a b$ & 10.57 & a & 10.19 & A \\
\hline Means & 9.76 & $\mathrm{D}$ & 9.94 & $\mathrm{C}$ & 10.11 & B & 10.18 & B & 10.43 & A & 10.51 & A & & \\
\hline \multicolumn{15}{|c|}{ Second Season (2015) } \\
\hline Distilled water (control) & 9.70 & $f-k$ & 9.80 & $\mathrm{c}-\mathrm{k}$ & 10.00 & a-g & 10.03 & $a-f$ & 10.17 & $a b$ & 10.20 & $\mathrm{a}$ & 9.98 & A \\
\hline $1 \mathrm{mM} \mathrm{SNP}$ & 9.60 & $\mathrm{ijk}$ & 9.87 & $\mathrm{a}-\mathrm{i}$ & 9.77 & $\mathrm{~d}-\mathrm{k}$ & 9.87 & $a-i$ & 10.00 & $a-g$ & 9.97 & $a-h$ & 9.84 & B \\
\hline $10 \mathrm{mM} \mathrm{OX}$ & 9.57 & $\mathrm{ijk}$ & 9.87 & $\mathrm{a}-\mathrm{i}$ & 9.80 & $\mathrm{c}-\mathrm{k}$ & 9.83 & $b-j$ & 10.03 & $a-f$ & 10.00 & $a-g$ & 9.85 & B \\
\hline $2 \% \mathrm{H}_{2} \mathrm{O}_{2}$ & 9.60 & ijk & 9.70 & $\mathrm{f}-\mathrm{k}$ & 9.83 & $b-j$ & 9.90 & $\mathrm{a}-\mathrm{i}$ & 10.07 & a-e & 10.07 & a-e & 9.86 & B \\
\hline $1 \mathrm{mM} \mathrm{SNP}+10 \mathrm{mM} \mathrm{OX}$ & 9.47 & $\mathrm{k}$ & 9.67 & $\mathrm{~g}-\mathrm{k}$ & 9.77 & d-k & 9.83 & $b-j$ & 10.03 & $a-f$ & 10.10 & a-d & 9.81 & B \\
\hline $1 \mathrm{mM} \mathrm{SNP}+2 \% \mathrm{H}_{2} \mathrm{O}_{2}$ & 9.50 & $\mathrm{jk}$ & 9.63 & $\mathrm{~h}-\mathrm{k}$ & 9.73 & $e-k$ & 9.77 & $\mathrm{~d}-\mathrm{k}$ & 10.03 & $a-f$ & 10.07 & a-e & 9.79 & B \\
\hline $10 \mathrm{mM} \mathrm{OA}+2 \% \mathrm{H}_{2} \mathrm{O}_{2}$ & 9.57 & $\mathrm{ijk}$ & 9.77 & $\mathrm{~d}-\mathrm{k}$ & 9.87 & $a-i$ & 9.87 & $a-i$ & 10.00 & $a-g$ & 10.13 & $a b c$ & 9.87 & B \\
\hline Means & 9.57 & $\mathrm{D}$ & 9.76 & $\mathrm{C}$ & 9.82 & $\mathrm{BC}$ & 9.87 & B & 10.05 & A & 10.08 & A & & \\
\hline
\end{tabular}

Means followed by the same letters within postharvest treatments, storage periods and their interactions in each season are not significantly different at level $P \leq 0.05$ according to DMRT.

Moreover, it is clear that, the postharvest treatment with the $1 \mathrm{mM}$ SNP individual or in combination with $10 \mathrm{mM} \mathrm{OA}$ or $2 \% \mathrm{H}_{2} \mathrm{O}_{2}$ gave the most effective influence on fruit content of SSC. While in case of titratable acidity and $\mathrm{SSC} / \mathrm{TA}$ ratio it is clear that, the treatments of $1 \mathrm{mM}$ SNP in combination with $2 \% \mathrm{H}_{2} \mathrm{O}_{2}$ followed by the other two combinations had the most effective in this aspect.

In case of ascorbic acid content changes, it is clear that, all combinations postharvest treatments had the most effective in this aspect and reduced the deterioration rate of 'Valencia' orange fruits content in AsA as compared to individual and control treatments during the first season. While during the second season, postharvest application of $1 \mathrm{mM} \mathrm{SNP}$ in combination with $10 \mathrm{mM}$ OA were superior in this aspect followed by postharvest treatments by the other two combinations. 
NITRIC OXIDE OXALIC ACID AND HYDROGEN PEROXIDE ...

TABLE 12. Influence of sodium nitroprusside (SNP), oxalic acid (OA) and hydrogen peroxide $\left(\mathrm{H}_{2} \mathrm{O}_{2}\right)$ on TA ( $\mathrm{g}$ citric acid/100 $\mathrm{ml}$ juice) of 'Valencia' oranges during storage at $8^{\circ} \mathrm{C}$ followed by one week shelf life at $18-23^{\circ} \mathrm{C}$

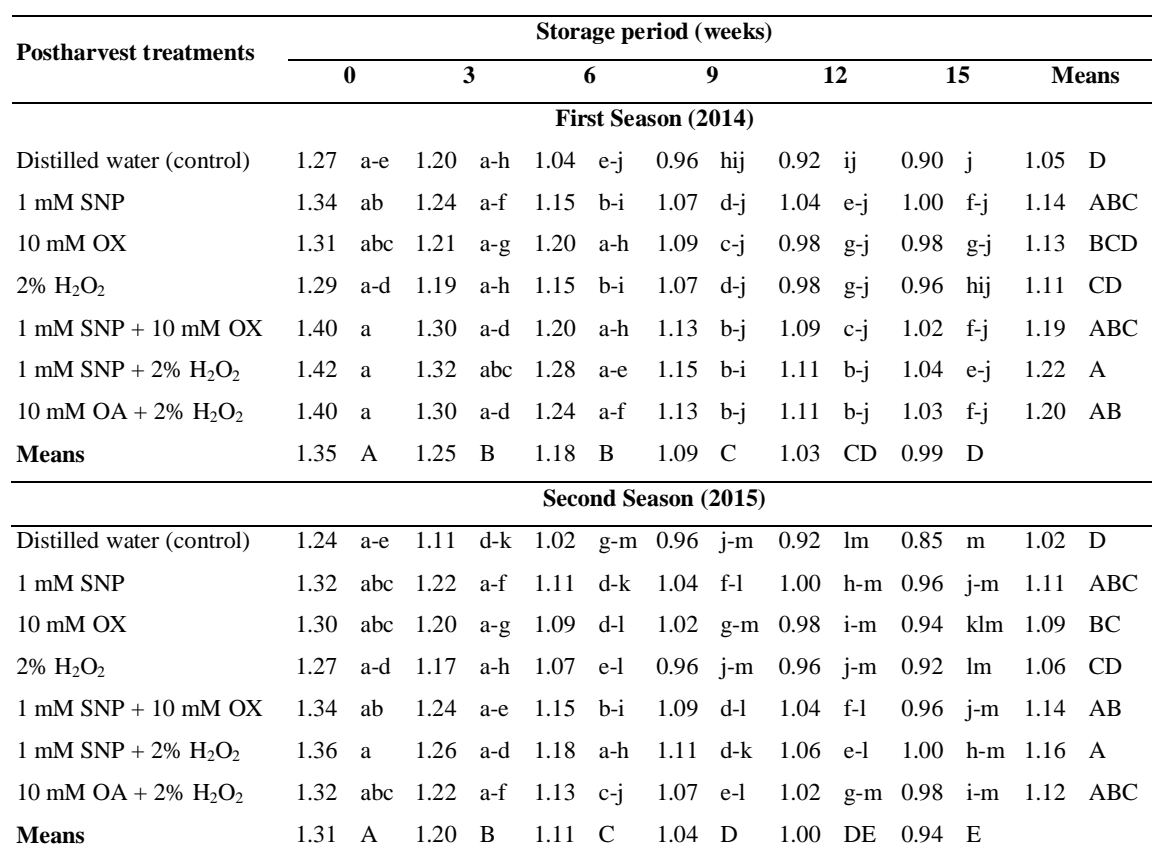

Means followed by the same letters within postharvest treatments, storage periods and their interactions in each season are not significantly different at level $P \leq 0.05$ according to DMRT.

OA treatment could be suppressed the lipid peroxidation and ultimately decreased the ascorbic acid oxidation (Kayashima \& Katayama, 2002). $\mathrm{H}_{2} \mathrm{O}_{2}$ treatment have beneficial effects on fruit physiology such as delaying ripening of oranges by the increasing antioxidants content in fruits (Saltveit and Sharaf, 1992).

The stability of ascorbic acid directly increased in the presence of $\mathrm{H}_{2} \mathrm{O}_{2}$ during storage of orange, grape and pomegranate fruit juices (Özkan et al. 2004 and Bayoumi, 2008).

In addition, the highest value of SSC in untreated fruits may be due to the increasing of water loss (Troncoso et al., 2005 and Bayoumi, 2008). Moreover, the decreasing juice content of TA with prolonging of cold storage period might be due to the degradation of citric acid during storage or their conversion into sugars and further utilization in metabolic process in the fruit (Rathore et al., 2007). 
TABLE 13. Influence of sodium nitroprusside (SNP), oxalic acid (OA) and hydrogen peroxide $\left(\mathrm{H}_{2} \mathrm{O}_{2}\right)$ on SSC/TA ratio of 'Valencia' oranges during storage at $8^{\circ} \mathrm{C}$ followed by one week shelf life at $18-23^{\circ} \mathrm{C}$

\begin{tabular}{|c|c|c|c|c|c|c|c|c|c|c|c|c|c|c|}
\hline \multirow{2}{*}{ Postharvest treatments } & \multicolumn{12}{|c|}{ Storage period (weeks) } & \multirow{2}{*}{\multicolumn{2}{|c|}{ Means }} \\
\hline & \multicolumn{2}{|c|}{$\mathbf{0}$} & \multicolumn{2}{|c|}{3} & \multicolumn{2}{|c|}{6} & \multicolumn{2}{|c|}{9} & \multicolumn{2}{|c|}{12} & \multicolumn{2}{|c|}{15} & & \\
\hline \multicolumn{15}{|c|}{ First Season (2014) } \\
\hline Distilled water (control) & 7.78 & $\mathrm{i}-1$ & 8.34 & f-1 & 9.61 & $b-i$ & 10.58 & $a-d$ & 11.52 & $a b$ & 11.75 & a & 9.93 & A \\
\hline $1 \mathrm{mM} \mathrm{SNP}$ & 7.28 & $\mathrm{kl}$ & 8.03 & h-1 & 8.73 & $d-1$ & 9.57 & $b-i$ & 10.39 & $a-f$ & 10.81 & $a-d$ & 9.14 & $\mathrm{BC}$ \\
\hline $10 \mathrm{mM} \mathrm{OX}$ & 7.45 & $\mathrm{jkl}$ & 8.23 & $\mathrm{~g}-1$ & 8.49 & e-l & 9.46 & $b-j$ & 10.78 & $a-d$ & 10.88 & $a b c$ & 9.22 & $\mathrm{ABC}$ \\
\hline $2 \% \mathrm{H} 2 \mathrm{O} 2$ & 7.56 & $\mathrm{i}-1$ & 8.36 & $f-1$ & 8.91 & $\mathrm{c}-1$ & 9.64 & $b-i$ & 10.67 & $a-d$ & 10.98 & $\mathrm{abc}$ & 9.35 & $\mathrm{AB}$ \\
\hline $1 \mathrm{mM} \mathrm{SNP}+10 \mathrm{mM} \mathrm{OX}$ & 6.95 & 1 & 7.64 & i-l & 8.49 & e-1 & 9.06 & $\mathrm{c}-\mathrm{k}$ & 9.61 & b-i & 10.32 & $\mathrm{a}-\mathrm{g}$ & 8.68 & $\mathrm{BC}$ \\
\hline $1 \mathrm{mM} \mathrm{SNP}+2 \% \mathrm{H} 2 \mathrm{O} 2$ & 6.87 & 1 & 7.54 & i-1 & 7.89 & i-1 & 8.88 & $\mathrm{c}-1$ & 9.40 & $c-j$ & 10.12 & $\mathrm{a}-\mathrm{h}$ & 8.45 & $\mathrm{C}$ \\
\hline $10 \mathrm{mM} \mathrm{OA}+2 \% \mathrm{H} 2 \mathrm{O} 2$ & 6.97 & $\mathrm{kl}$ & 7.66 & $\mathrm{i}-1$ & 8.25 & $\mathrm{~g}-1$ & 9.57 & $b-i$ & 9.48 & $b-j$ & 10.49 & a-e & 8.74 & $\mathrm{BC}$ \\
\hline \multirow[t]{2}{*}{ Means } & 7.27 & $\mathrm{E}$ & 7.97 & $\mathrm{D}$ & 8.62 & $\mathrm{C}$ & 9.54 & $\mathrm{~B}$ & 10.26 & $\mathrm{~A}$ & 10.76 & A & & \\
\hline & \multicolumn{12}{|c|}{ Second Season (2015) } & & \\
\hline Distilled water (control) & 7.85 & $\mathrm{~m}-\mathrm{r}$ & 8.87 & $f-p$ & 9.79 & b-k & 10.48 & a-g & 11.17 & $\mathrm{ab}$ & 11.96 & $\mathrm{a}$ & 10.02 & $\mathrm{~A}$ \\
\hline $1 \mathrm{mM}$ SNP & 7.31 & pqr & 8.13 & k-r & 8.85 & f-p & 9.44 & $\mathrm{c}-\mathrm{m}$ & 10.03 & $b-j$ & 10.61 & a-e & 9.06 & $\mathrm{BCD}$ \\
\hline $10 \mathrm{mM} \mathrm{OX}$ & 7.39 & o-r & 8.26 & k-r & 9.03 & e-o & 9.62 & b-1 & 10.29 & b-h & 10.85 & $a-d$ & 9.24 & $\mathrm{BC}$ \\
\hline $2 \% \mathrm{H} 2 \mathrm{O} 2$ & 7.77 & m-r & 8.55 & i-r & 9.23 & d-n & 10.35 & $\mathrm{a}-\mathrm{h}$ & 10.52 & $a-g$ & 11.03 & $a b c$ & 9.58 & $\mathrm{AB}$ \\
\hline $1 \mathrm{mM} \mathrm{SNP}+10 \mathrm{mM} \mathrm{OX}$ & 7.11 & $\mathrm{qr}$ & 7.85 & m-r & 8.49 & i-r & 9.07 & e-o & 9.61 & b-1 & 10.55 & $a-f$ & 8.78 & $\mathrm{CD}$ \\
\hline $1 \mathrm{mM} \mathrm{SNP}+2 \% \mathrm{H} 2 \mathrm{O} 2$ & 7.02 & $\mathrm{r}$ & 7.69 & $\mathrm{n}-\mathrm{r}$ & 8.32 & $j-r$ & 8.81 & g-p & 9.44 & $\mathrm{c}-\mathrm{m}$ & 10.07 & b-i & 8.56 & $\mathrm{D}$ \\
\hline $10 \mathrm{mM} \mathrm{OA}+2 \% \mathrm{H} 2 \mathrm{O} 2$ & 7.28 & pqr & 8.05 & $1-r$ & 8.75 & h-q & 9.31 & d-n & 9.82 & b-k & 10.34 & $a-h$ & 8.92 & $\mathrm{CD}$ \\
\hline Means & 7.39 & $\mathrm{~F}$ & 8.20 & $\mathrm{E}$ & 8.92 & $\mathrm{D}$ & 9.58 & $\mathrm{C}$ & 10.13 & B & 10.77 & A & & \\
\hline
\end{tabular}

Means followed by the same letters within postharvest treatments, storage periods and their interactions in each season are not significantly different at level $P \leq 0.05$ according to DMRT.

Based on these explanation could be suggested that, postharvest treatments especially $1 \mathrm{mM}$ SNP in combination with $10 \mathrm{mM} \mathrm{OA}$ or $2 \% \mathrm{H}_{2} \mathrm{O}_{2}$ applications increased fruit juice contents of AsA and TA and decrease of SSC as compared to untreated fruits during cold storage period at $8^{\circ} \mathrm{C}$ followed by one week at ambient temperature.

Our results are in accordance the findings of Zheng et al. (2007b, c), Zaharah \& Singh (2011a, b) and Razzaq et al. (2015) on mangoes, Sayyari et al. (2010) on pomegranates and $\mathrm{Li}$ et al. (2014) on papaya fruits. They claimed that, postharvest application of NO and OA reduced fruit content of SSC and SSC/TA ratio as well as increased TA and AsA as compared to untreated fruits during cold storage.

Egypt. J. Hort. Vol. 43, No. 1 (2016) 
NITRIC OXIDE OXALIC ACID AND HYDROGEN PEROXIDE ...

TABLE 14. Influence of sodium nitroprusside (SNP), oxalic acid (OA) and hydrogen peroxide $\left(\mathrm{H}_{2} \mathrm{O}_{2}\right)$ on AsA (mg/100 ml juice) of 'Valencia' oranges during storage at $8^{\circ} \mathrm{C}$ followed by one week shelf life at $18-23^{\circ} \mathrm{C}$

\begin{tabular}{|c|c|c|c|c|c|c|c|}
\hline \multirow{2}{*}{ Postharvest treatments } & \multicolumn{6}{|c|}{ Storage period (weeks) } & \multirow[b]{2}{*}{ Means } \\
\hline & $\mathbf{0}$ & 3 & 6 & 9 & 12 & 15 & \\
\hline \multicolumn{8}{|c|}{ First Season (2014) } \\
\hline Distilled water (control) & $63.61 \mathrm{a}-\mathrm{f}$ & 58.18 h-m & 51.52 qrs & $47.57 \mathrm{t}$ & $42.12 \mathrm{u}$ & $37.27 \mathrm{v}$ & $50.05 \mathrm{D}$ \\
\hline $1 \mathrm{mM}$ SNP & $65.52 \mathrm{abc}$ & $61.52 \mathrm{c}-\mathrm{h}$ & $58.79 \mathrm{~g}-1$ & 56.67 j-p & 54.24 m-r & $51.21 \mathrm{q}-\mathrm{t}$ & $57.99 \mathrm{C}$ \\
\hline $10 \mathrm{mM} \mathrm{OX}$ & 65.21 a-d & $61.21 \mathrm{~d}-\mathrm{h}$ & 57.57 h-o & 56.06 k-p & 53.94 n-r & 50.91 rst & $57.48 \mathrm{C}$ \\
\hline $2 \% \mathrm{H}_{2} \mathrm{O}_{2}$ & 64.91 a-e & 60.91 e-i & 56.67 j-p & $55.15 \quad \mathrm{l}-\mathrm{q}$ & $52.73 \mathrm{p}-\mathrm{s}$ & $49.09 \mathrm{st}$ & $56.58 \mathrm{C}$ \\
\hline $1 \mathrm{mM} \mathrm{SNP}+10 \mathrm{mM} \mathrm{OX}$ & 66.85 a & 64.85 a-e & 62.73 a-g & $61.52 \mathrm{c}-\mathrm{h}$ & $59.09 \mathrm{~g}-1$ & 56.66 j-p & $61.95 \mathrm{~A}$ \\
\hline $1 \mathrm{mM} \mathrm{SNP}+2 \% \mathrm{H}_{2} \mathrm{O}_{2}$ & $66.55 \mathrm{ab}$ & 64.55 a-e & $62.42 \mathrm{~b}-\mathrm{g}$ & $60.31 \mathrm{f}-\mathrm{j}$ & 57.88 h-n & $54.55 \mathrm{~m}-\mathrm{r}$ & $61.04 \mathrm{AB}$ \\
\hline $10 \mathrm{mM} \mathrm{OA}+2 \% \mathrm{H}_{2} \mathrm{O}_{2}$ & $65.94 \mathrm{ab}$ & 63.94 a-f & $62.43 \mathrm{~b}-\mathrm{g}$ & $59.39 \mathrm{~g}-\mathrm{k}$ & 56.97 i-o & 53.64 o-r & $60.39 \mathrm{~B}$ \\
\hline Means & $65.51 \mathrm{~A}$ & $62.17 \mathrm{~B}$ & $58.88 \mathrm{C}$ & $56.67 \mathrm{D}$ & $53.85 \mathrm{E}$ & $50.48 \mathrm{~F}$ & \\
\hline \multicolumn{8}{|c|}{ Second Season (2015) } \\
\hline Distilled water (control) & 62.81 a-d & $57.58 \mathrm{~g}-1$ & $52.420-\mathrm{s}$ & $46.36 \mathrm{t}$ & $40.30 \mathrm{u}$ & $36.06 \mathrm{v}$ & $49.26 \mathrm{E}$ \\
\hline $1 \mathrm{mM}$ SNP & $63.94 \mathrm{abc}$ & 60.61 b-h & $57.88 \mathrm{~g}-\mathrm{k}$ & 55.46 j-p & $53.941-q$ & 50.91 qrs & $57.12 \mathrm{C}$ \\
\hline $10 \mathrm{mM} \mathrm{OX}$ & $64.03 \mathrm{abc}$ & $59.70 \mathrm{~d}-\mathrm{i}$ & 56.36 i-n & $54.24 \mathrm{k}-\mathrm{q}$ & 53.03 n-r & $49.39 \mathrm{rst}$ & $56.13 \mathrm{CD}$ \\
\hline $2 \% \mathrm{H} 2 \mathrm{O} 2$ & $63.74 \mathrm{abc}$ & $59.09 \mathrm{~d}-\mathrm{j}$ & 56.06 i-o & $53.941-q$ & $52.12 \mathrm{p}-\mathrm{s}$ & $48.79 \mathrm{st}$ & $55.62 \mathrm{D}$ \\
\hline $1 \mathrm{mM} \mathrm{SNP}+10 \mathrm{mM}$ OX & 65.94 a & $63.94 \mathrm{abc}$ & 62.12 a-f & $60.30 \mathrm{c}-\mathrm{h}$ & $58.48 \mathrm{f}-\mathrm{j}$ & 56.37 i-n & $61.19 \mathrm{~A}$ \\
\hline $1 \mathrm{mM} \mathrm{SNP}+2 \% \mathrm{H} 2 \mathrm{O} 2$ & $64.43 \mathrm{ab}$ & 62.43 a-e & $61.21 \mathrm{~b}-\mathrm{g}$ & $58.79 \mathrm{e}-\mathrm{j}$ & 56.97 h-m & $53.34 \mathrm{~m}-\mathrm{q}$ & $59.53 \mathrm{~B}$ \\
\hline $10 \mathrm{mM} \mathrm{OA}+2 \% \mathrm{H} 2 \mathrm{O} 2$ & $64.12 \mathrm{abc}$ & 62.12 a-f & $60.30 \mathrm{c}-\mathrm{h}$ & $57.88 \mathrm{~g}-\mathrm{k}$ & 55.45 j-p & $52.43 \mathrm{o}-\mathrm{s}$ & $58.72 \mathrm{~B}$ \\
\hline Means & $64.14 \mathrm{~A}$ & 60.78 B & $58.05 \mathrm{C}$ & $55.28 \mathrm{D}$ & $52.90 \mathrm{E}$ & $49.61 \mathrm{~F}$ & \\
\hline
\end{tabular}

Means followed by the same letters within postharvest treatments, storage periods and their interactions in each season are not significantly different at level $P \leq 0.05$ according to DMRT.

In conclusion, postharvest application of 'Valencia' orange fruits with $1 \mathrm{mM}$ SNP, $10 \mathrm{mM} \mathrm{OA}$ and $2 \% \mathrm{H}_{2} \mathrm{O}_{2}$ especially $1 \mathrm{mM}$ SNP in combination with 10 $\mathrm{mM}$ OA or $2 \% \mathrm{H}_{2} \mathrm{O}_{2}$ treatments controlled decay incidence and reduced fruit weight loss percentage with increased marketable percentage as well as maintained fruit firmness. Moreover, these applications reduced the activities of PPO and PE enzymes besides enhanced the activity of POX enzyme with maintained the inner fruit quality during cold storage at $8^{\circ} \mathrm{C}$ and $85-95 \% \mathrm{RH}$ for up to 15 weeks.

Author contributions: A.F. Abd El-khalek conceived of study, designed the experiment and purchased the chemicals. A.F. Abd El-khalek and H.G. Elmehrat performed the experiment. Gehan. A. Mahmoud determined enzyme activities only. A.F. Abd El-khalek analyzed the data and wrote the manuscript. M.A.A Mohamed revised the manuscript.

Conflicts of interest: the authors declare that there are no conflicts of interest related to the publication of this study. 


\section{References}

Afek, U., Orenstein, J. and Nuriel, E. (1999) Fogging disinfectants inside storage room against pathogens of potatoes and sweet potatoes. Crop Protection, 18, 111-114.

AOAC, (1990) "Official Methods of Analysis of the Association of Official Analytical Chemists", $15^{\text {th }}$ ed., Association of Official Analytical Chemists, Arlington VA, pp. 1058-1059.

Bayoumi, A.Y. (2008) Improvement of postharvest keeping quality of white pepper fruits (Capsicum annuum, L.) by hydrogen peroxide treatment under storage conditions. Acta Biologica Szegediensis, 52 (1), 7-15.

Cheng, G., Yang, E., Lu, W., Jia, Y., Jiang, Y. and Duan, X. (2009) Effect of nitric oxide on ethylene synthesis and softening of banana fruit slice during ripening. Journal of Agricultural and Food Chemistry, 57, 5799-5804.

Collinge, B., Kragh, K.M., Mikkelsen, J.D., Nielsen, K.K., Rasmussen, U. and Vad, K. (1993) Plant chitinases. Plant Journal, 3, 31-40.

Crawford, N.M. (2006) Mechanisms for nitric oxide synthesis in plants. Journal of Experimental Botany, 57, 471-478.

Ding, Z.S., Tian, S., Zheng, X.L., Zhou, Z.W. and Xu, Y. (2007) Responses of reactive oxygen metabolism and quality in mango fruit to exogenous oxalic acid or salicylic acid under chilling temperature stress. Physiol. Plant, 130, 112-121.

Fernandez S., Ribeiro, C., Raposo, M., Morais, R. and Morais, A. (2011) Polyphenol Oxidase Activity and Colour Changes of 'Starking' Apple Cubes Coated with Alginate and Dehydrated with Air. Food and Nutrition Sciences, 2 (5), 451-457.

Horwitz, W., Senzel, A., Reynolds, H. and Park, D.L. (1975) Official Methods of Analysis of the Association of Official Analytical Chemists. $12^{\text {th }}$ ed., $1094 \mathrm{p}$.

Huang, H., Q. Zhu, Z. Zhang, B. Yang, B., X. Duan and Y. Jiang (2013b) Effect of oxalic acid on anti browning of banana (Musa spp., AAA group, cv. 'Brazil') fruit during storage. Scientia Horticulturae, 160, 208-212.

Huang, H., Jing, G., Guo, L., Zhang, D., Yang, B., Duan, X., Ashraf, M. and Jiang, Y. (2013a) Effect of oxalic acid on ripening attributes of banana fruit during storage. Postharvest Biology and Technology, 84, 22-27.

Jin, P., Zhu, H., Wang, L., Shan, T.M. and Zheng, Y.H. (2014) Oxalic acid alleviates chilling injury in peach fruit by regulating energy metabolism and fatty acid contents. Food Chemistry, 161, 87-93.

Kang, R., Zhang, L., Jiang, L., Yu, M., Ma, R. and Yu, Z. (2016) Effect of postharvest nitric oxide treatment on the proteome of peach fruit during ripening. Postharvest Biology and Technology, 112, 277-289.

Kayashima, T. and Katayama, T. (2002) Oxalic acid is available as a natural antioxidant in some systems. Biochim. Biophys. Acta, 1573, 1-3.

Egypt. J. Hort. Vol. 43, No. 1 (2016) 
Ku, V.V.V., Wills, R.B.H. and Leshem, Y.Y. (2000) Use of nitric oxide to reduce postharvest water loss from horticultural produce. Journal of Horticultural Science and Biotechnology, 75, 268-270.

Lawton, K.A., Friedrich, L., Hunt, M., Weymann, K., Delaney, T., Kessmann, H., Staub, T. and Ryals, J. (1996) Benzothiadiazole induces disease resistance in Arabidopsis by activation of the systemic acquired resistance signal transduction pathway. The Plant Journal, 10, 71-82.

Leshem, Y.Y., Wills, R.B.H. and Ku, V.V. (1998) Evidence for the function of the free radical gas nitric oxide (NO-) as an endogenous maturation and senescence regulating factor in higher plants. Plant Physiology and Biochemistry, 36, 825-833.

Li, X.P., Wu, B., Guo, Q., Wang, J.D., Zhang, P. and Chen, W.X. (2014) Effect of nitric oxide on postharvest quality and soluble sugar content in papaya fruit during ripening. J. Food Process, 38 (1), 591-599.

Manjunatha, G., Lokesh, V. and Neelwarne, B. (2010) Nitric oxide in fruit ripening: trends and opportunities. Biotechnology Advanced, 28, 489-499.

McGuire, R.G. (1992) Reporting of objective colour measurements. HortScience, 27 (12), 1254-1255.

M-STAT, (1993) A microcomputer program for the design, arrangement and analysis of agronomic research experiments. Michigan State University.

Mucharromah, E. and Kuc, J. (1991) Oxalate and phosphates induce systemic resistance against diseases caused by fungi, bacteria and viruses in cucumber. Crop Protection, 10 (4), 265-270.

Őzkan, M., Krca, A. and Cemeroglu, B. (2004) Effects of hydrogen peroxide on the stability of ascorbic acid during storage in various fruit juices. Food Chemistry, $\mathbf{8 8}$ (4), 591-597.

Rathore, H. A., Masud, T., Sammi, S. and Soomro, H. A. (2007) Effect of storage on physico-chemical composition and sensory properties of mango (Mangifera indica L.) variety Dosehari. Pakistan Journal of Nutrition, 6 (2), 143-148.

Razzaq, K., Khan, A.S., Malik, A.U., Shahid, M. and Ullah, S. (2015) Effect of oxalic acid application on Samar Bahisht Chauns amango during ripening and postharvest. Food Science and Technology, 63, 152- 160.

Saltveit, M.E. and Sharaf, A.R. (1992) Ethanol inhibitors ripening of tomato fruit harvested at various degrees of ripeness without affecting subsequent quality. Journal of the American Society for Horticultural Science, 117, 793-798.

Sapers, G.M and Simmons G.F. (1998) Hydrogen peroxide disinfections of minimally processed fruits and vegetables. Food Technology, 52 (2), 48-52. 
Sapers, G.M, Miller, R.L., Pilizota, V. and Kamp, F. (2001) Shelf life extension of fresh mushrooms (Agaricus bisporus) by application of hydrogen peroxide and browning inhibitors. Journal of Food Science, 66, 362-366.

Sayyari, M., Valero, D., Babalar, M., Kalantari, S., Zapata, P.J. and Serrano, M. (2010) Prestorage oxalic acid treatment maintained visual quality, bioactive compounds, and antioxidant potential of pomegranate after long-term storage at $2^{\circ} \mathrm{C}$. Journal of Agricultural and Food Chemistry, 58, 6804-6808.

Schlumbaum, A., Mauch, F., Vogeli, U. and Boller, T. (1986) Plant chitinases are potent inhibitors of fungal growth. Nature, 324, 365-367.

Singh, S.P., Singh, Z. and Swinny, E.E. (2009) Postharvest nitric oxide fumigation delays fruit ripening and alleviates chilling injury during cold storage of Japanese plums (Prunus salicina Lindell). Postharvest Biology and Technology, 53, 101-108.

Smilanick, J.L., Margosan, D.A. and Henson, D.J. (1995) Evaluation of heated solutions of sulfur dioxide, ethanol, and hydrogen peroxide to control postharvest green mold of lemons. Plant Dis., 79, 742-747.

Steel, R.G.D., Torrie, J.H. and Diskey, D.A. (1997) "Principles and Procedures of Statistics. A Biometrical Approach", $3^{\text {rd }}$ ed., McGrow-Hill Publishing Company, New York, USA, 336-376.

Suslow, T. (1997) Postharvest chlorination: Basic properties and key points for effective disinfection. Agricultural and Natural Resources Dept, California Univ., Oakland, CA 94612-3560, (510), 987-1096.

Tian, S.P., Wan, Y.K., Qin, G.Z. and Xu, Y. (2006) Induction of defense responses against Alternaria rot by different elicitors in harvested pear fruit. Applied Microbiology and Biotechnology, 70, 729-734.

Toal, E.S. and Jones P.W. (1999) Induction of systemic resistance to Sclerotinia sclerotiorum by oxalic acid in oilseed rape. Plant Pathology, 48, 759-767.

Troncoso, R., Espinoza, C., Sánchez-Estrada, A., Tiznado, M.E. and Hugo, S.G. (2005) Analysis of the isothiocyanates present in cabbage leaves extract and their potential application to control Alternaria rot in bell peppers. Food Res. Inter., 38, 701-708.

Ueda, M., Sasaki, K., Utsunomiya, N. and Shimabayashi, Y. (2001) Changes in properties during maturation and ripening of 'Chiin Hwang No. 1' mango fruit cultivated in a plastic greenhouse. Food Science and Technology Research, 7, 207213.

Valero, D. and Serrano, M. (2010) "Postharvest Biology and Technology for Preserving Fruit Quality", CRC-Taylor and Francis, Boca Raton, USA.

Voss, D.H. (1992) Relating colourimeter measurement of plant colour to the royal horticultural society colour chart. HortScience, 27 (12), 1256-1260. 
Wang, Z., Cao, J. and Jiang, W. (2016) Changes in sugar metabolism caused by exogenous oxalic acid related to chilling tolerance of apricot fruit. Postharvest Biology and Technology, 14, 10-16.

Watkins, C. and Harman, J. (1981) Use of penetrometer to measure flesh firmness of fruit. Orchadist, N. Z., 14-16.

Wills, R.B.H., Soegiarto, L. and Bowyer, M.C. (2007) Use of a solid mixture containing diethylenetriamine/nitric oxide (DETANO) to liberate nitric oxide gas in the presence of horticultural produce to extend postharvest life. Nitric Oxide, 17, 44-49.

Wisniewski, M.E. and Wilson, C.L. (1992) Biological control of postharvest diseases of fruits and vegetables: recent advances. HortScience, 27, 94-98.

Wu, F., Yang, H., Chang, Y., Cheng, J., Bai, S. and Yin, J. (2012) Effects of nitric oxide on reactive oxygen species and antioxidant capacity in Chinese Bayberry during storage. Scientia Horticulturae, 135, 106-111.

Wu, F., Zhang, D., Zhang, H., Jiang, G., Su, X., Qu, H., Jiang, Y. and Duan, X. (2011) Physiological and biochemical response of harvested plum fruit to oxalic acid during ripening or shelf life. Food Res. Inter., 44, 1299-1305.

Yang, E., Yang, S.Y., Lu, W.J., Chen, J.Y., Qu, H.X., Jiang, Y., Joyce, D.C. and Duan, X.W. (2010) Effect of nitric oxide on the sensitivity of banana fruit slices to ethylene. J. Hort. Sci. Biotechnology, 85, 17-22.

Yao, H.J. and Tian, S.P. (2005) Effects of pre and post-harvest application of salicylic acid or methyl jasmonate on inducing disease resistance of sweet cherry fruit in storage. Postharvest Biology and Technology, 35, 253-262.

Yoruk, R., Balaban, M.O., Marshall, M.R. and Yoruk, S. (2002) The inhibitory effect of oxalic acid on browning of banana slices. In: Annual Meeting and Food Expo, Anaheim, California, 30 G-18, p.74.

Zaharah, S.S. and Singh, Z. (2011a) Postharvest nitric oxide fumigation alleviates chilling injury, delays fruit ripening and maintains quality in cold stored 'Kensington Pride' mango. Postharvest Biology and Technology, 60, 202-210.

Zaharah, S.S. and Singh, Z. (2011b) Mode of action of nitric oxide in inhibiting ethylene biosynthesis and fruit softening during ripening and cool storage of 'Kensington Pride' mango. Postharvest Biology and Technology, 62, 258-266.

Zhang, D.D., Cheng, G.P., Li, J., Yang, E., Qu, H.X., Jiang, Y.M. and Duan, X.M. (2008) Effect of nitric oxide on disorder development and quality maintenance of plum fruit stored at low temperature. Acta Horticultural, 804, 549-554.

Zheng, X. and Tian, S. (2006) Effect of oxalic acid on control of postharvest browning of litchi fruit. Food Chemist., 96, 519-523.

Zheng, X., Tian, S., Meng, X. and Li, B. (2007a) Physiological and biochemical responses in peach fruit to oxalic acid treatment during storage at room temperature. Food Chemistry, 104, 156-162. 
Zheng, X.L., Tian, S.P., Gidley, M.J., Yue, H. and Li, B.Q. (2007b) Effects of exogenous oxalic acid on ripening and decay incidence in mango fruit during storage at room temperature. Postharvest Biology and Technology, 45, 281-284.

Zheng, X., Ye, L., Jiang, T., Jing, G. and Li, J. (2012) Limiting the deterioration of mango fruit during storage at room temperature by oxalate treatment. Food Chemistry, 130, 279-285.

Zheng, X.L., Tian, S.P., Gidley, M.J., Yue, H., Li, B.Q. and Xu, Y. (2007c) Slowing deterioration of mango fruit during cold storage by prestorage application of oxalic acid. J. Hort. Sci. Biotechnology, 82 (5), 707-714.

Zhu, S.H. and Zhou, J. (2007) Effect of nitric oxide on ethylene production in strawberry fruit during storage. Food Chemistry, 100, 1517-1522.

Zhu, S.H., Liu, M.C. and Zhou, J. (2006) Inhibition by nitric oxide of ethylene biosynthesis and lipoxygenase activity in peach fruit during storage. Postharvest Biology and Technology, 42, 41-48.

Zhu, S.H., Sun, L. and Zhou, J. (2010a) Effects of different nitric oxide application on quality of kiwifruit during $20^{\circ} \mathrm{C}$ storage. International Journal of Food Science and Technology, 45, 245-251.

Zhu, L.Q., Zhou, J. and Zhu, S.H. (2010b) Effect of a combination of nitric oxide treatment and intermittent warming on prevention of chilling injury of 'Feicheng' peach fruit during storage. Food Chemistry, 121, 165-170. 


\section{المعاملة بأكسيد النيتريك وحامض الأوكساليك وفئ أكسيد

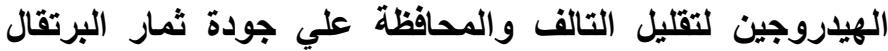 الفالنشيا أثناء التخزين المبرد \\ محمود على أحمد محمد" ، أحمد فتحي عبد الخالق"**، حسن جابر المحرات*** ،

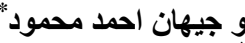

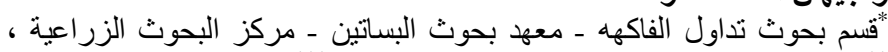

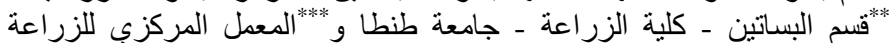 العضوية ـ مركز البحوث الزر اعية ـ القاهرة ـ مصر.}

تم دراسة تأثير معاملات ما بعد الحصاد بأكسيد النيتريك وحامض الأوكساليك الكيك

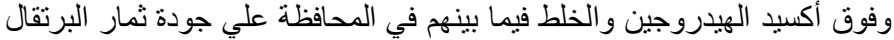

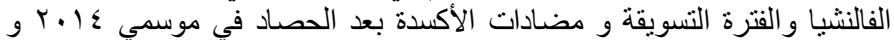

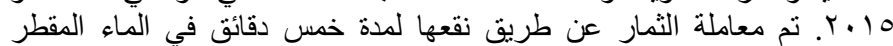

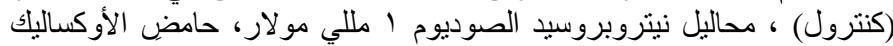

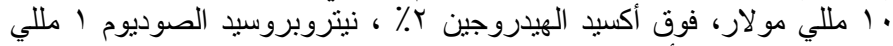

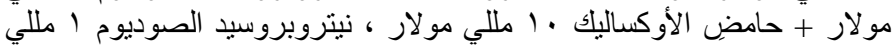

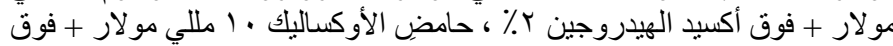

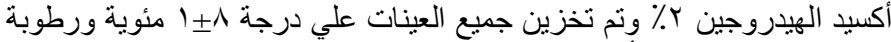

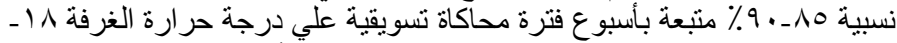

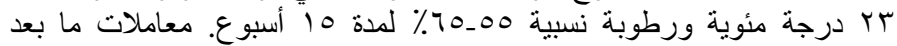

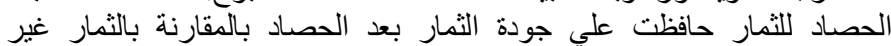

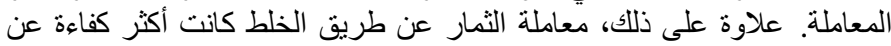

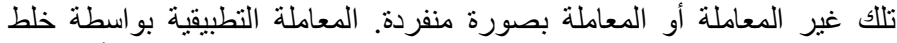

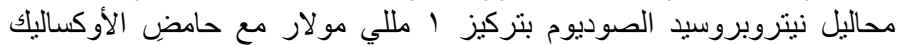

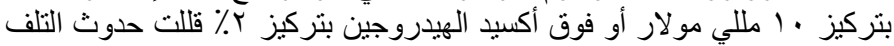

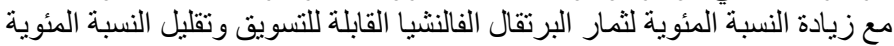

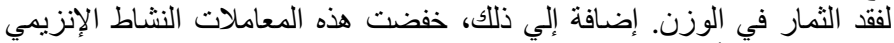

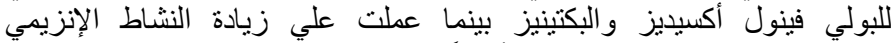

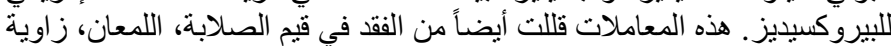

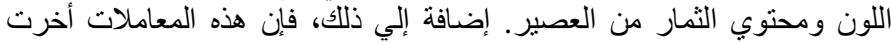

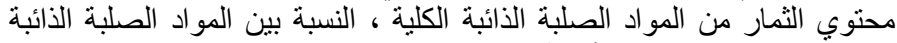

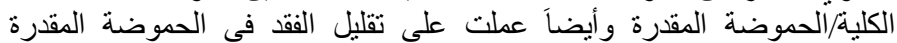

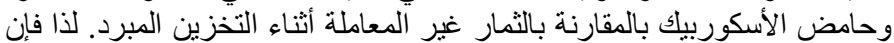

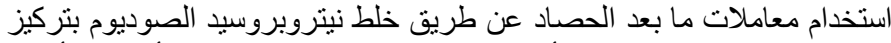

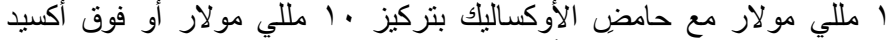

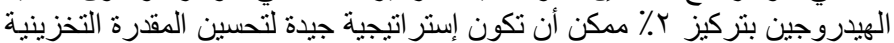
وتقليل التلف الحادث وأيضاً المحافظة علي جودة ثمار البرتئن البقال الفالنشيا أثناء

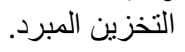

